\title{
miR-137 regulates ferroptosis by targeting glutamine transporter SLC1A5 in melanoma
}

\author{
Meiying Luo ${ }^{1} \cdot$ Longfei $\mathrm{Wu}^{2} \cdot$ Kexin $\mathrm{Zhang}^{1} \cdot$ Hong Wang $^{3} \cdot$ Tian Zhang $^{4} \cdot$ Lucas Gutierrez $^{4} \cdot$ Douglas O'Connell $^{5}$. \\ Peng Zhang ${ }^{1} \cdot \mathrm{Yu} \mathrm{\textrm {Li } ^ { 6 }} \cdot$ Tongtong $\mathrm{Gao}^{1} \cdot$ Wenyan $\mathrm{Ren}^{7} \cdot$ Yongfei Yang $\mathbb{1}^{1}$
}

Received: 9 August 2017 / Revised: 23 November 2017 / Accepted: 5 December 2017 / Published online: 18 January 2018

(c) The Author(s) 2018. This article is published with open access

\begin{abstract}
Ferroptosis is a regulated form of cell death driven by small molecules or conditions that induce lipid-based reactive oxygen species (ROS) accumulation. This form of iron-dependent cell death is morphologically and genetically distinct from apoptosis, necroptosis, and autophagy. miRNAs are known to play crucial roles in diverse fundamental biological processes. However, to date no study has reported miRNA-mediated regulation of ferroptosis. Here we show that miR-137 negatively regulates ferroptosis by directly targeting glutamine transporter SLC1A5 in melanoma cells. Ectopic expression of miR-137 suppressed SLC1A5, resulting in decreased glutamine uptake and malondialdehyde (MDA) accumulation. Meanwhile, antagomir-mediated inactivation of endogenous miR-137 increased the sensitivity of melanoma cells to erastin- and RSL3induced ferroptosis. Importantly, knockdown of miR-137 increased the antitumor activity of erastin by enhancing ferroptosis both in vitro and in vivo. Collectively, these data indicate that miR-137 plays a novel and indispensable role in ferroptosis by inhibiting glutaminolysis and suggest a potential therapeutic approach for melanoma.
\end{abstract}

\section{Introduction}

Edited by R.A. Knight

Meiying Luo, Longfei Wu, and Kexin Zhang contributed equally to this work.

Electronic supplementary material The online version of this article (https://doi.org/10.1038/s41418-017-0053-8) contains supplementary material, which is available to authorized users.

Yongfei Yang

yangyf@bit.edu.cn

1 School of Life Science, Beijing Institute of Technology, Beijing 100081, China

2 Center for Genetic Epidemiology and Genomics, School of Public Health, Soochow University, Suzhou, Jiangsu 215123, China

3 Department of Pharmacy, State Key Laboratory of Pathogens and Biosecurity, Beijing Institute of Microbiology and Epidemiology, Beijing 100071, China

4 School of Pharmacy, University of Southern California, Los Angeles, CA 90033, USA

5 Department of Medicine, UC Irvine School of Medicine, Orange, CA 92697, USA

6 Laboratory of Vaccine and Antibody Engineering, Beijing Institute of Biotechnology, Beijing 100071, China

7 Cam-Su Genomic Resource Center, Soochow University, Suzhou 215123, China
Cell death plays an important role in a variety of contexts, such as maintaining homeostasis during development and disease prevention [1-3]. Previously, it was assumed that apoptosis was the only regulated form of cell death [2, 3]. However, this view has been recently challenged by the discovery of several nonapoptotic cell death pathways [46], including necroptosis [7] and ferroptosis [8].

Distinct from apoptosis, necrosis, and autophagy, ferroptosis is an oxidative, iron-dependent form of cell death [8-10]. The ferroptosis-inducing compounds, eradicator of Ras and ST (erastin) [11] and Ras selective lethal 3 (RSL3) [12], were discovered using high-throughput screening of small-molecule libraries. The cell death induced by these compounds was triggered by inactivation of cellular glutathione (GSH)-dependent antioxidant defenses, leading to the accumulation of toxic lipid reactive oxygen species (ROS) [8-10, 13], and notably was absent of apoptotic hallmarks [11-13]. Specifically, erastin inhibits the glutamate (Glu)/cystine antiporter of system $\mathrm{x}_{\mathrm{c}}{ }^{-}$and consequently, the import of cystine. A lack of cystine, an important precursor of GSH synthesis, results in the reduced level of GSH and ROS accumulation [8]. In addition, RSL3 directly binds and inhibits glutathione peroxidase 4 (GPX4), 
which is a critical antioxidant enzyme that can reduce lipid hydroperoxides within biological membranes [9, 14]. Without adequate levels of GPX4, GSH cannot function as a reducing agent within the local peroxidase reaction cycle and thus causes an accumulation of lipid ROS and induction of ferroptosis. Both erastin and RSL3 share this common cell death mechanism, which causes loss of cellular antioxidant capacity that leads to ferroptosis $[8,15]$.

Recently, additional genes and pathways have been found to modulate ferroptosis, including iron metabolism, lipid metabolism, and amino-acid metabolism [10, 16-19]. L-glutamine (Gln) has long been known to be essential for cancer cell growth. Recent studies have demonstrated that Gln metabolism contributes to the formation of oxidizable lipids to induce ferroptosis [10, 20]. The Gln importer SLC1A5/SLC38A1, glutaminases 2 and glutamicoxaloacetic transaminase 1 (GOT1) are required for Gln uptake and metabolism to Glu and ultimately to aketoglutarate (a-KG). Knockdown of these genes provided cell partial immunity to ferroptosis induction [10].

MicroRNAs (miRNAs) are a class of endogenously expressed, 22 nucleotides (nt) non-coding RNAs, which post-transcriptionally regulate gene expression. Importantly, miRNAs play an essential role in a broad range of biological processes including proliferation, differentiation, apoptosis, and autophagy, linking them to numerous diseases including cancer [21-23]. However, no miRNAs have been reported to directly regulate ferroptosis so far. Following an unbiased screen of miRNAs affecting erastin- and RSL3-induced ferroptosis, we discovered that miR-137 suppressed ferroptosis by directly targeting the Gln importer SLC1A5. Our findings underline the importance of miRNA in ferroptosis regulation and introduce miR-137 as an important regulator of ferroptosis in melanoma.

\section{Materials and methods}

\section{Cell culture and transfection}

Melanoma cell lines A375 and G-361 were obtained from American Type Culture Collection (ATCC, USA) and cultured in Dulbecco's modified Eagle's medium (DMEM) supplemented with $10 \%$ fetal bovine serum (Invitrogen), $\quad 2 \mathrm{mM}$ L-glutamine and $1 \%$ penicillin-streptomycin (Gibco-BRL). Cells were dissociated with $0.05 \%$ trypsin and counted with an automated cell counter ( $\mu$ Scope Cell Counter Basic, Xietong ChenDong Tech., China). Transfections were performed according to the manufacturer's instructions with Lipofectamine 2000 (Invitrogen) or RNAiMax transfection reagent (Invitrogen).

\section{Plasmids}

To generate miR-137 overexpression constructs, a 361-bp fragment up and downstream of the pre-miR-137 was amplified from HEK293T complementary DNA (cDNA) by PCR (forward primer, 5'-GCTCAGCGAGCAGCAAGAGT-3' and reverse primer, 5'-GGCAATAAGAGCGAAACACCA-3'), and cloned into pcDNA5/FRT/ TO vector with $K p n I$ and XhoI restriction enzyme sites or pCDH-CMV-MCS-EF1-Puro vector with XbaI and BamHI restriction enzyme sites. The anti-miR-137 and antiScramble oligos were obtained from Genepharma (Shanghai, China). To generate stable and specific knockdown miR-137 vector, we cloned miR-137 target sequence $\left(5^{\prime}\right.$ TACAGACCATCATTTAGCAATAC-3') into pCDHCMV-MCS-EF1-Puro lentiviral vector with $X b a I$ and BamHI restriction enzyme sites. The full-length cDNA of human SLC1A5 was cloned from HEK293T cDNA by PCR. The 3'-UTR regions of SLC1A5 and SLC38A1 were amplified by PCR and then cloned into psiCHECK-2 Vector (Promega) (SLC1A5 forward 5'-CAGGGAGCAGGTCACAGGTC-3', and SLC1A5 reverse 5'CAGTTTTCAGACTGCAGCAAATC-3'; SLC38A1 forward, 5'-CCAATTCAGTGAGGCACAGTGTT-3' and SLC38A1 reverse primer, 5'-CGTAAATTTGAGCCTGTATACAG-3'). To generate the $3^{\prime}$-UTR mutants of SLC1A5, the binding site (AGCAAUCC) was changed to (CAACCAAU) using the site-directed mutagenesis kit (NEB E0554) and verified by sequencing.

\section{Antibodies and chemicals}

The following antibodies were used in this study: SLC1A5 (ab84903, Abcam), glutaminase 2 (GLS2; ab113509, Abcam), GOT1 (PA5-24634, Thermo), SLC38A1 (ab59721, Abcam), Flag (F3165, clone M2; SigmaAldrich), Actin (sc-8432, Santa Cruz). HRP-labeled secondary antibody conjugates were purchased from Molecular Probes (Thermo). Erastin (\#E7781), RSL3 (\#S8155), and ferrostatin-1 (\#S7243) were obtained from Selleck Chemicals (Houston, TX, USA). Necrosulfonamide (ab143839) and Z-VAD-FMK (ab120382) were obtained from Abcam. Compound 968 (352010), GPNA (G1135), and aminooxyacetate (AOA; C13408) were obtained from Sigma.

\section{Immunoblotting}

For immunoblotting, cells were washed with ice-cold phosphate-buffered saline (PBS), lysed in lysis buffer (20 $\mathrm{mM}$ Tris at $\mathrm{pH} 7.5,150 \mathrm{mM} \mathrm{NaCl}, 1 \mathrm{mM}$ EDTA, and $2 \%$ Triton X-100), supplemented with a phosphatase inhibitor mix (Pierce) and a complete protease inhibitor cocktail (Roche). Cell lysates were resolved by sodium dodecyl 
sulfate-polyacrylamide gel electrophoresis and transferred to a polyvinylidene difluoride membrane (Bio-Rad). Membranes were blocked with $5 \%$ non-fat milk, and probed with the indicated antibodies. Horseradish peroxidaseconjugated goat secondary antibodies were used (1:5000, Invitrogen). Immunodetection was achieved with the Hyglo chemiluminescence reagent (Denville Scientific), and detected by a Bio-Rad ECL machine.

\section{Luciferase reporter assay}

Melanoma cells were cultured in six-well plates and cotransfected with a psiCHECK-2 luciferase reporter plasmid and either anti-miR-137 or miR-137 overexpression construct. Cells were lysed $48 \mathrm{~h}$ after transfection and assayed with the dual-luciferase reporter assay system (Promega E1910), and measurements were made on the BeckmanCoulter DTX880. At least four replicates with three independent experiments were performed, transfection efficiency was normalized using Renilla luciferase.

\section{RNA extraction, cDNA synthesis, and real-time PCR analysis}

Total RNA was isolated with the RNeasy Mini Kit (Qiagen 74104 ), and $1 \mu \mathrm{g}$ of total RNA was used for cDNA synthesis using the iScript ${ }^{\text {TM }}$ cDNA Synthesis Kit (Bio-Rad). Quantitative real-time PCRs were carried out using iQ SYBR Green Master Mix (Bio-Rad). Samples were obtained and analyzed on the CFX96 Touch Real-Time PCR Detection System. The gene expression levels were normalized to actin. The primer sequences used for PCR were: SLC1A5 forward, 5'-CAACCTGGTGTCAGCAGCCTT-3' and SLC1A5 reverse, 5'-GCACCGTCCATGTTGACGGTG-3'; SLC38A1 forward, 5'CCTCCTATTGATCTGTTCAA-3' and SLC38A1 reverse, 5'-TTAGCTCTGGAACAATGCAG-3'; actin forward, 5'GCTCGTCGTCGACAACGGCT-3' and actin reverse, 5'CAAACATGATCTGGCTCATCTTCTC-3'. To verify the expression of miR-137, total RNA was prepared using the RNeasy Mini Kit (Qiagen 74104), and $1 \mu \mathrm{g}$ of total RNA was used for cDNA synthesis using TaqMan ${ }^{\mathrm{TM}}$ MicroRNA Reverse Transcription Kit (Thermo 4366596). The quantitative PCR analysis was performed with TaqMan miRNA assays and normalized to small nuclear RNA (Rnu6) (Thermo 4426961).

\section{Cell viability assay}

Cell viability was evaluated using the Cell Counting Kit-8 (CCK-8) (\#96992, Sigma) according to the manufacturer's instructions. The assay is based on utilizing the highly water-soluble tetrazolium salt WST-8 [2-(2-methoxy-4- nitrophenyl)-3-(4-nitrophenyl)-5- (2,4-disulfophenyl)-2Htetrazolium,monosodium salt] to produce a water-soluble formazan dye upon reduction in the presence of an electron carrier. Absorbance at $450 \mathrm{~nm}$ is proportional to the number of living cells in the culture.

\section{Colony formation assay}

For the colony formation assay, cells were seeded in $60 \mathrm{~mm}$ dishes. At 70-80\% confluence, cells were treated with indicated drugs for $24 \mathrm{~h}$. Cells were trypsinized, counted, and re-plated in appropriate dilutions for colony formation. After 10-14 days of incubation, colonies were fixed and stained in a mixture of $6 \%$ glutaraldehyde (Amresco) and $0.5 \%$ crystal violet, carefully rinsed with tap water, and dried at room temperature. Plating efficiency was determined for each individual cell line and the surviving fraction was calculated based on the number of colonies that arise after treatment. Each experiment was repeated three times.

\section{Malondialdehyde (MDA) assay}

The relative MDA concentration in cell lysates was assessed using a Lipid Peroxidation Assay Kit (\#ab118970, Abcam) according to the manufacturer's instructions. Briefly, the MDA in the sample reacted with thiobarbituric acid (TBA) to generate a MDA-TBA adduct. The MDA-TBA adduct was quantified colorimetrically (optical density (OD) $=532 \mathrm{~nm})$.

\section{Iron assay}

Intracellular ferrous iron $\left(\mathrm{Fe}^{2+}\right)$ level was determined using the iron assay kit purchased from Abcam and was used according to the manufacturer's instructions. Briefly, samples were collected and washed in cold PBS. Samples were homogenized in $5 \mathrm{X}$ volumes of iron assay buffer on ice. The supernatant was collected and iron reducer was added to each sample before mixing, and incubating for $30 \mathrm{~min}$. Then, the iron probe was added to each sample before mixing, and incubating for $60 \mathrm{~min}$. The output was measured immediately on a colorimetric microplate reader (optical density (OD) $593 \mathrm{~nm}$ ).

\section{Glutamine uptake assay}

The $\left[{ }^{3} \mathrm{H}\right]$-L-glutamine uptake was performed as described previously [24, 25]. Briefly, cells were cultured in six-well plates in glutamine-free DMEM/F-12 medium (Invitrogen). After collecting and counting, cells were incubated with $200 \mathrm{nM}\left[{ }^{3} \mathrm{H}\right]$-L-glutamine (PerkinElmer) in glutamine-free $\mathrm{DMEM} / \mathrm{F}-12$ medium for $15 \mathrm{~min}$ at $37^{\circ} \mathrm{C}$ in the presence or 

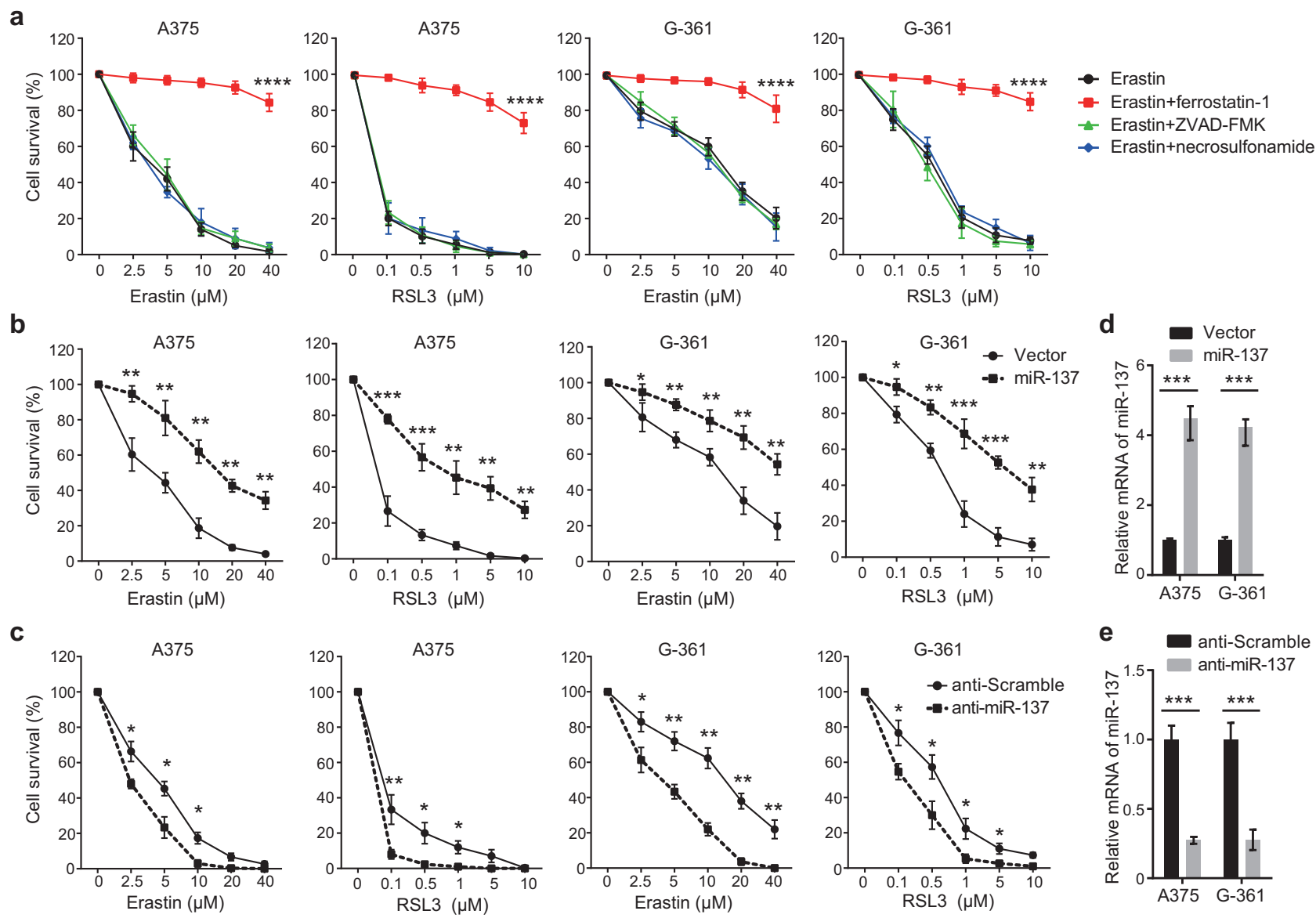

Fig. 1 miR-137 negatively regulates ferroptosis in melanoma. a The A375 and G-361 human melanoma cells were treated with erastin $(2.5-40 \mu \mathrm{M})$ or RSL3 $(0.1-10 \mu \mathrm{M})$ with or without a cell death inhibitor (ferrostatin-1, $1 \mu \mathrm{M}$; ZVAD-FMK, $10 \mu \mathrm{M}$; necrosulfonamide, $0.5 \mu \mathrm{M})$ for $24 \mathrm{~h}$. Cell death was assayed using a CCK-8 kit. Data shown represent mean \pm SD from three independent experiments. $* * * * p<0.0001$. b Overexpression of miR-137 suppressed erastin- and RSL3-induced cell death in human melanoma cells. Indicated cells were treated with erastin $(2.5-40 \mu \mathrm{M})$ or RSL3 $(0.1-10 \mu \mathrm{M})$ for $24 \mathrm{~h}$. Cell death was assayed using a CCK-8 kit. Data shown represent mean \pm SD from three independent experiments. $* p<0.05$; $* * p<$

absence of erastin and RSL3. Cells were collected, transferred to filter paper using a 96-well plate harvester (Wallac PerkinElmer), dried, exposed to scintillation fluid, and counts measured using a liquid scintillation counter (PerkinElmer).

\section{Lipid ROS assay using flow cytometer}

Lipid ROS level was determined using $5 \mu \mathrm{M}$ of BODIPYC11 dye purchased from Thermo according to previous works $[9,26]$. Briefly, cells were seeded in six-well plates. The next day, culture medium was replaced with $2 \mathrm{ml}$ medium containing $5 \mu \mathrm{M}$ of BODIPY-C11 and the culture was returned to the cell culture incubator for $20 \mathrm{~min}$. Cells were harvested in $15 \mathrm{ml}$ tubes and washed twice with PBS
$0.01 ; * * * p<0.001$. c Inhibition of endogenous miR-137 enhanced erastin- and RSL3-induced cell death in human melanoma cells. Indicated cells were treated with erastin $(2.5-40 \mu \mathrm{M})$ or RSL3 $(0.1-10$ $\mu \mathrm{M})$ for $24 \mathrm{~h}$. Cell death was assayed using a CCK-8 kit. Data shown represent mean \pm SD from three independent experiments. ${ }^{*} p<0.05$; $* * p<0.01$. d Quantitative RT-PCR showing the relative expression levels of miR-137 in b. Data are mean \pm SD from three independent experiments. $* * * p<0.001$. e Quantitative RT-PCR showing the relative expression levels of miR-137 in d. Data are mean \pm SD from three independent experiments. $* * * p<0.001$

followed by re-suspending in $500 \mu \mathrm{l}$ of PBS. The cell suspension was filtered through cell strainer $(0.4 \mu \mathrm{m}$ nylon mesh) and subjected to the flow cytometry analysis to examine the amount of ROS within cells. The fluorescence intensities of cells per sample were determined by flow cytometry using the BD FACSAria cytometer (BD Biosciences).

\section{shRNA constructs}

All shRNAs were purchased from Sigma. The shRNA clones IDs are the following: SLC38A1 KD: TRCN0000069231; GLS2 KD: TRCN0000177027, SLC1A5 KD: TRCN0000340676; GOT1 KD: TRCN0000119795. 

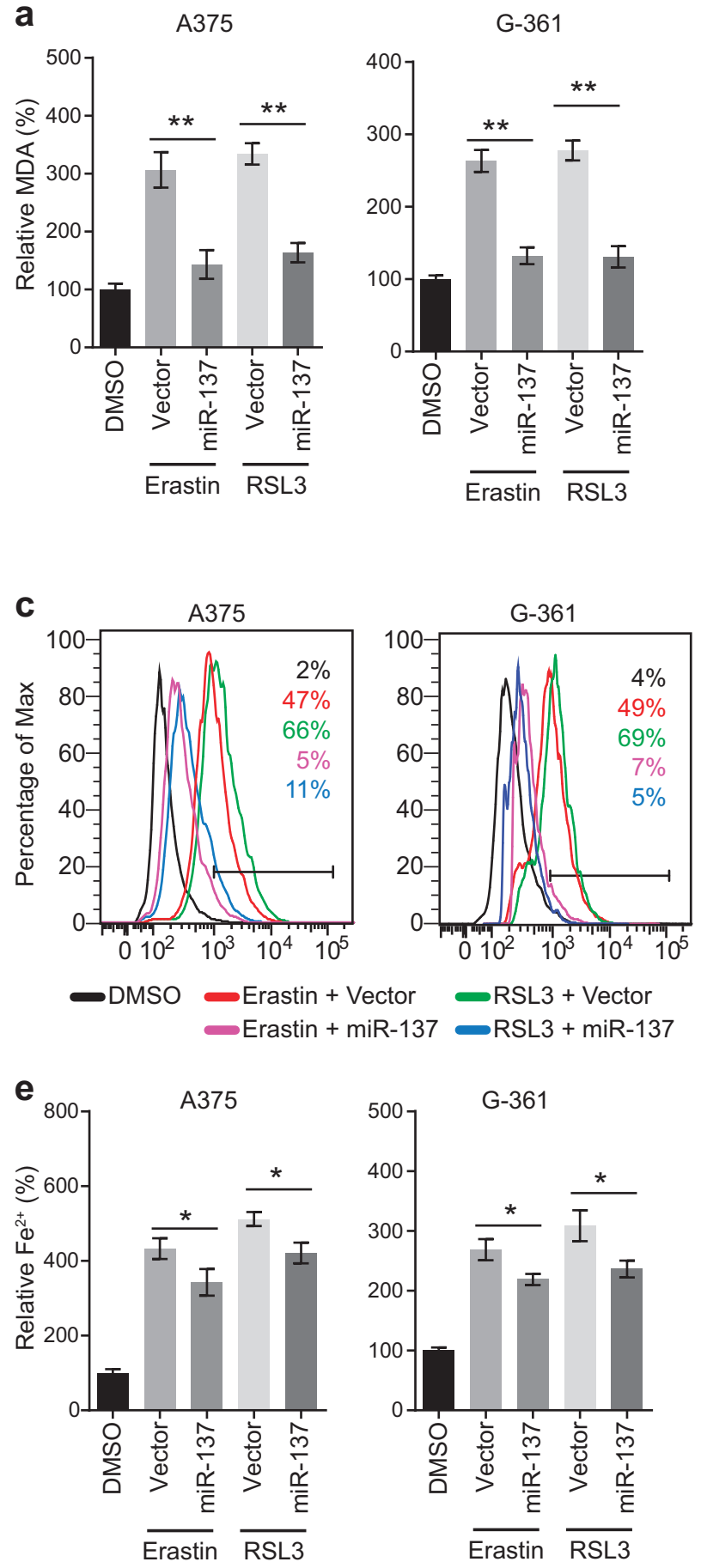

Fig. 2 miR-137 regulates the lipid metabolism of melanoma cells. a miR-137 overexpression significantly suppressed the erastin- and RSL3induced lipid formation. Indicated cells were treated with erastin $(5 \mu \mathrm{M}$ in $\mathrm{A} 375,10 \mu \mathrm{M}$ in G-361) or RSL3 (0.1 $\mu \mathrm{M}$ in $\mathrm{A} 375,0.5 \mu \mathrm{M}$ in $\mathrm{G}-361)$ for $24 \mathrm{~h}$. The lipid formation was measured by MDA assay. Data shown represent mean \pm SD from three independent experiments. $* * p<0.01$. b miR-137 antagomirs significantly increased the erastin- and RSL3induced lipid formation. Indicated cells were treated with erastin $(5 \mu \mathrm{M}$ in $\mathrm{A} 375,10 \mu \mathrm{M}$ in G-361) or RSL3 $(0.1 \mu \mathrm{M}$ in A375, $0.5 \mu \mathrm{M}$ in G-361) for $24 \mathrm{~h}$. The lipid formation was measured by MDA assay. Data shown represent mean \pm SD from three independent experiments. ${ }^{* *} p<0.01$. c Overexpression of miR-137 significantly suppressed erastin- and RSL3- b
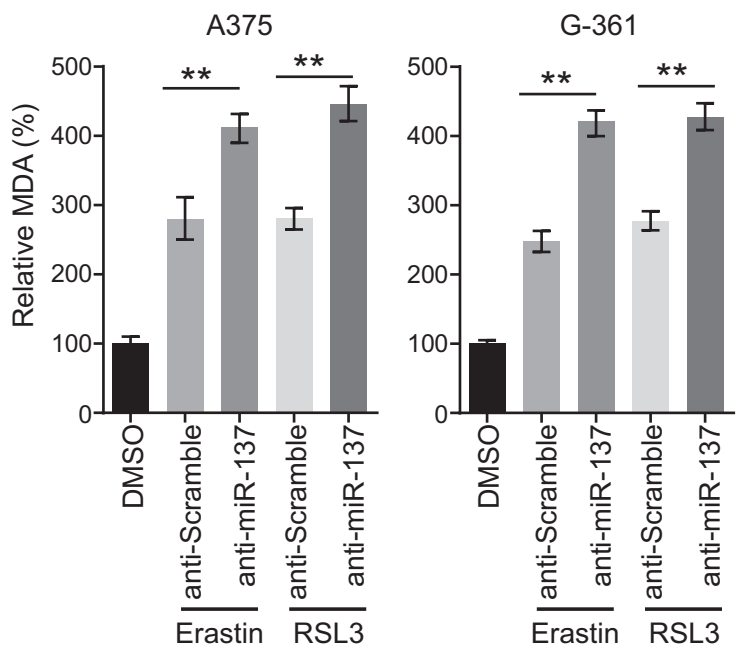

d
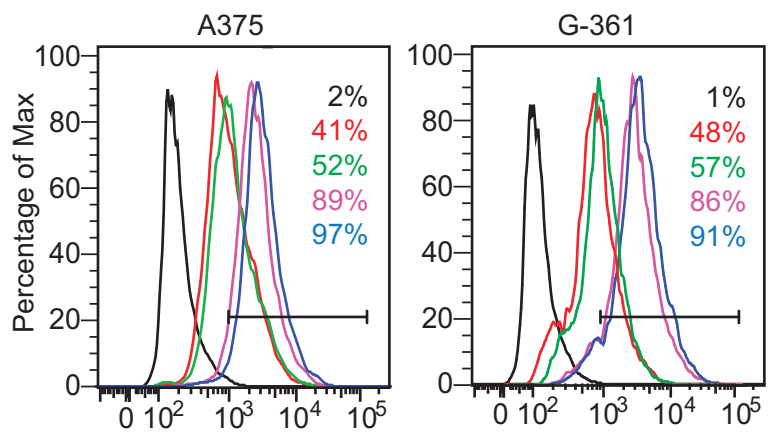

-DMSO f

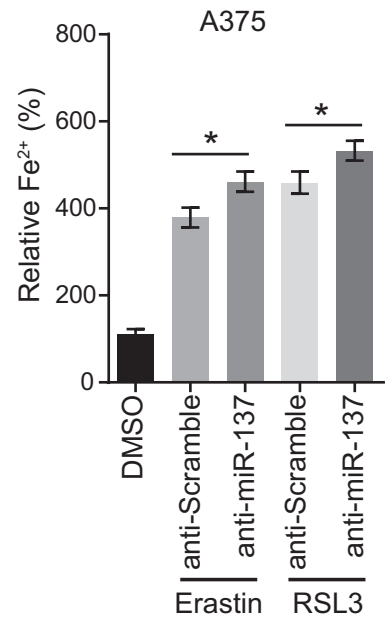

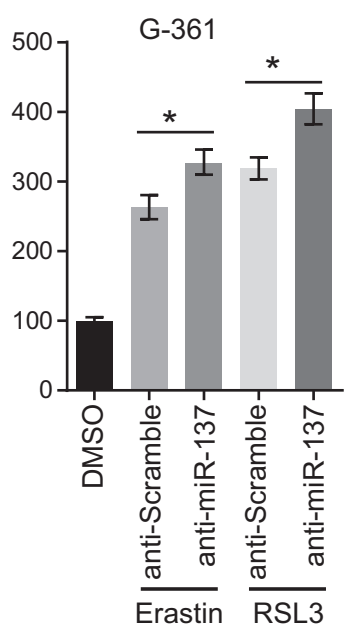

induced lipid ROS level as assessed by flow cytometry using C11BODIPY. Indicated cells were treated with erastin $(5 \mu \mathrm{M}$ in A375, 10 $\mu \mathrm{M}$ in G-361) or RSL3 $(0.1 \mu \mathrm{M}$ in A375, $0.5 \mu \mathrm{M}$ in G-361) for $24 \mathrm{~h}$. d Cells transfected with miR-137 antagomirs showed increased lipid ROS level as assessed by BODIPY-C11 staining. Indicated cells were treated with erastin $(5 \mu \mathrm{M}$ in A375, $10 \mu \mathrm{M}$ in G-361) or RSL3 $(0.1 \mu \mathrm{M}$ in A375, $0.5 \mu \mathrm{M}$ in G-361) for $24 \mathrm{~h}$. e, f The expression levels of miR-137 influences the $\mathrm{Fe}^{2+}$ accumulation in erastin or RSL3-treated A375 and G-361 cells. Indicated cells were treated with erastin $(5 \mu \mathrm{M}$ in A375, 10 $\mu \mathrm{M}$ in G-361) or RSL3 ( $0.1 \mu \mathrm{M}$ in A375, $0.5 \mu \mathrm{M}$ in G-361) for $24 \mathrm{~h}$ and the intracellular $\mathrm{Fe}^{2+}$ was assayed. Data shown represent mean $\pm \mathrm{SD}$ from three independent experiments. ${ }^{*} p<0.05$ 


\section{Lentivirus production}

For lentivirus production, HEK293T cells were transfected with pLKO.1/puro plasmids together with pCMV-dR8.91 and pCMV-VSV-G packing plasmids using Calcium Phosphate Transfection Kits (Clontech). Viral particles were collected $48 \mathrm{~h}$ after transfection, filtered with $0.45 \mu \mathrm{m}$ sterile filter and concentrated by ultracentrifugation at $4{ }^{\circ} \mathrm{C}$ $(24,000 \mathrm{rpm}, 2 \mathrm{~h}$, Beckman-Coulter ultracentrifuge XL$100 \mathrm{~K})$.

\section{Xenograph mouse model}

To generate murine subcutaneous tumors, melanoma cells $\left(5 \times 10^{6}\right.$ cells per mouse $)$ were injected subcutaneously into the right posterior flanks of 7-week-old immunodeficient nude mice. Tumor formation was monitored and tumor volume based on calliper measurements was calculated by the modified ellipsoidal formula (tumor volume $=1 / 2$ $\left(\right.$ length $\times$ width $\left.^{2}\right)$ ). When tumors reached a volume of approximately $50 \mathrm{~mm}^{3}$, mice were randomly allocated into groups and treated with erastin for 20 days. The erastin was dissolved in 5\% dimethylsulfoxide (DMSO) + corn oil (C8267, Sigma). To better dissolve erastin, we warmed the tube at $37^{\circ} \mathrm{C}$ water base and shake it gently. All animal procedures were performed in accordance with institutional guidelines.

\section{Statistical analysis}

All experiments were independently repeated at least three times. Data were represented as mean \pm SD. Unpaired Student's $t$-tests were used to compare the means of two groups. One-way analysis of variance (ANOVA) was used for comparison among the different groups. When ANOVA was significant, post hoc testing of differences between groups was performed using the least significant difference test. All data were analyzed by GraphPad Prism 6.0 (GraphPad Software, Inc.). A $p$-value $<0.05$ was considered statistically significant.

\section{Results}

\section{miR-137 regulates erastin- and RSL3-induced ferroptosis in melanoma}

Erastin and RSL3 are two classical small-molecule inducers of ferroptosis, which were lethal at lower doses in engineered human tumor cells expressing oncogenic HRAS $^{\mathrm{V} 12}$ than in isogenic cells expressing wild-type HRAS [8]. Given the oncogenic RAS mutants increased basal ROS levels in fibroblasts through the RAS-RAF-MEK-MAPK pathway, we analyzed the ferroptosis induction activity of erastin and RSL3 in two human melanoma cell lines that harbor the $\mathrm{BRAF}^{\mathrm{V} 600 \mathrm{E}}$ mutation, $\mathrm{A} 375$ and $\mathrm{G}-361$. Both erastin $\left(\mathrm{IC}_{50} \quad(\mathrm{~A} 375)=3.52 \mu \mathrm{M} ; \mathrm{IC}_{50} \quad(\mathrm{G}-361)=\right.$ $11.83 \mu \mathrm{M})$ and RSL3 (IC $50 \quad(\mathrm{~A} 375)=0.074 \mu \mathrm{M} ; \quad \mathrm{IC}_{50}$ $(\mathrm{G}-361)=0.44 \mu \mathrm{M})$ induced cell death in A375 and G-361 cells in a dose-dependent manner, this process was reversed by the ferroptosis inhibitor ferrostatin-1 (Fig. 1a). However, the apoptosis inhibitor ZVAD-FMK and necroptosis inhibitor necrosulfonamide had no effects on both erastin- and RSL3-induced cell death (Fig. 1a). These results indicate that erastin and RSL3 can sufficiently induce ferroptosis in melanoma cells carrying $\mathrm{BRAF}^{\mathrm{V} 600 \mathrm{E}}$ mutation.

To discover miRNAs that regulates ferroptosis, we performed an unbiased screen in melanoma cells transfected with miRNA overexpressing constructs. After erastin and RSL3 treatment, cell death was analyzed by the CCK- 8 cell viability assay. One of the miRNAs blocking erastin- and RSL3-induced ferroptosis was identified as miR-137. Compared with the cells transfected with empty vector, the cell viability of miR-137-overexpressing cells was significantly increased (Fig. 1b), suggesting that miR-137 may be a negative regulator of ferroptosis. The chemically engineered oligonucleotides, termed "antagomirs", are efficient and specific silencers of endogenous miRNAs [27]. To further confirm the role of miR-137 in ferroptosis, we knocked down miR-137 by transfecting cells with miR-137 specific antagomir. As shown in Fig. 1c, suppression of miR-137 expression increased erastin- and RSL3-induced cell death in both A375 and G-361 cells. The cellular levels of miR-137 were significantly modulated by miR-137 overexpression construct (Fig. 1d) and anti-miR-137 oligonucleotides (Fig. 1e). These findings indicate that miR137 is an important regulator of ferroptosis in melanoma cells.

\section{miR-137 inhibits lipid peroxidation and iron accumulation in ferroptosis}

To explore the molecular mechanism of miR-137 in ferroptosis, we analyzed lipid peroxidation and iron accumulation, which are two important signaling events in triggering ferroptosis. Lipid peroxidation is indispensable for ferroptosis, and GPX4 prevents ferroptosis through clearance of lipid peroxides [9]. As MDA is one of the most important end-products of lipid peroxidation, we tested whether miR-137 regulates MDA accumulation in melanoma cells. As shown in Fig. 2a, the elevated levels of MDA induced by erastin and RSL3 were significantly suppressed by the overexpression of miR-137. Moreover, inhibition of endogenous miR-137 by antagomir increased the MDA accumulation in both A375 and G-361 cells 
a

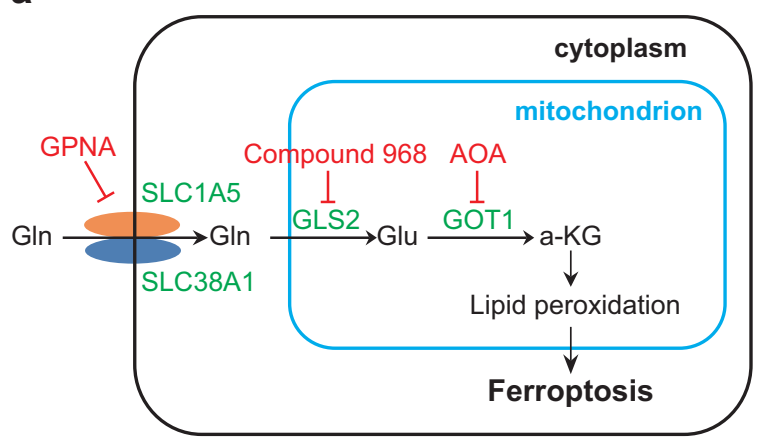

b

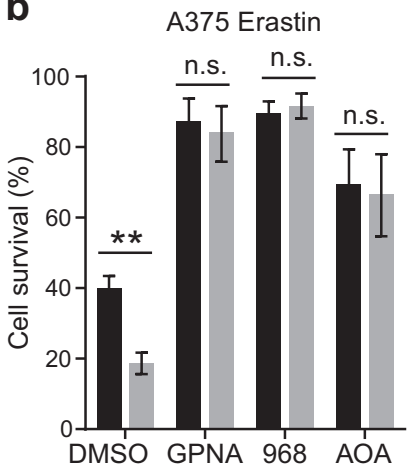

A375 Erastin

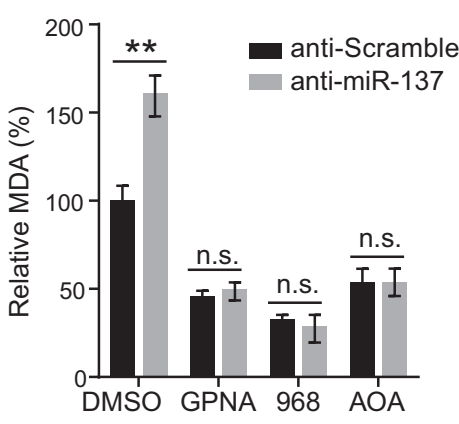

C

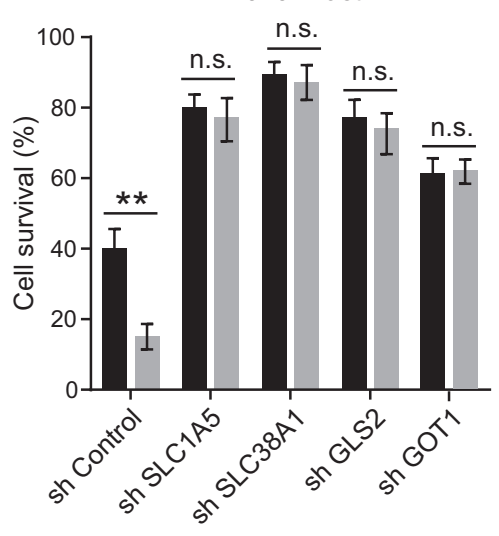

A375 Erastin

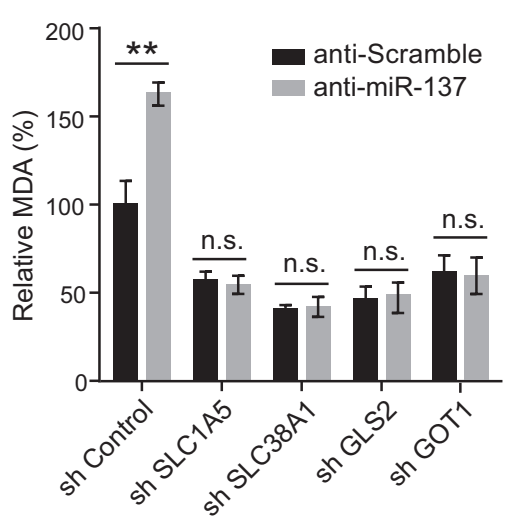

d

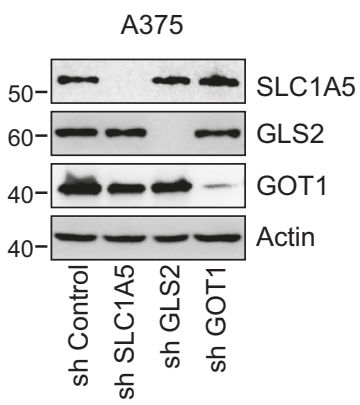

e

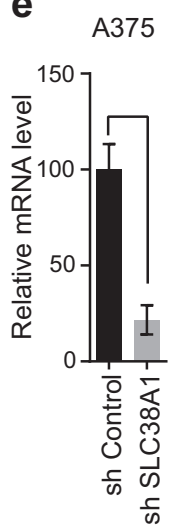

Fig. 3 Glutaminolysis mediates miR-137-induced ferroptosis inhibition. a Schematic overview of the glutaminolysis pathway in ferroptosis. Glutamine (Gln) is transferred into cell cytoplasm by importers SLC1A5/SLC38A1, and subsequently converted to glutamate (Glu) by glutaminase GLS2 in mitochondria. The aspartate transaminase GOT1 ultimately converts Glu to a-ketoglutarate (a-KG), which contributes to ROS accumulation by an unknown mechanism. The small-molecule inhibitors are indicated in red: L-Gln transporter inhibitor GPNA; GLS inhibitor Compound 968; Pan-transaminases inhibitor AOA. b Pharmacological inhibition of multiple components in the glutaminolysis pathway abrogated anti-miR-137-mediated ferroptosis cell death and lipid accumulation. Cells transfected with control or miR-137 antagomirs were treated with erastin $(5 \mu \mathrm{M})$ and GPNA $(5 \mathrm{mM}) /$ compound $968(20 \mu \mathrm{M}) / \mathrm{AOA}(0.5 \mathrm{mM})$ for $24 \mathrm{~h}$. The cell viability was assayed using a CCK- 8 kit and the lipid accumulation was measured by MDA assay. Data shown represent mean \pm SD from three independent experiments. n.s. nonsignificant; $* * p<0.01$. c Knockdown of Gln importers or metabolic enzymes blocked anti-miR-137-mediated ferroptosis cell death and lipid accumulation. Cells stably expressing control shRNA or indicated shRNA targeting metabolic enzymes were transfected with control or miR-137 antagomir, and then treated with erastin $(5 \mu \mathrm{M})$ for $24 \mathrm{~h}$. The cell viability was assayed using a CCK-8 kit and the lipid accumulation was measured by MDA assay. Data shown represent mean \pm SD from three independent experiments. ${ }^{* *} p$ $<0.01$. d The knockdown efficiency of three shRNA constructs targeting SLC1A5, GLS2 and GOT1 were detected by western blot. e The knockdown efficiency of shRNA construct targeting SLC38A1 was measured by real-time RT-PCR. Data shown represent mean \pm $\mathrm{SD}$ from three independent experiments. $* * * p<0.001$.
(Fig. 2b). Similarly, the lipid ROS generation assayed by flow cytometry using the fluorescent probes C11-BODIPY were suppressed by miR-137 overexpression in erastin and RSL3-treated cells (Fig. 2c). Knockdown of miR-137 induced lipid ROS generation in A375 and G-361 cells, as revealed by BODIPY-C11 staining (Fig. 2d).

Ferrous iron $\left(\mathrm{Fe}^{2+}\right)$ is another essential factor for the execution of ferroptosis. The iron chelators, such as DFO, CPX, 311 and 2,2-BP, can prevent cells from undergoing ferroptosis, whether induced by erastin, RSL3, or a physiological stimulus, such as high concentrations of extracellular Glu [8, 9, 12]. Therefore, we further investigated the effect of miR-137 on the changes of $\mathrm{Fe}^{2+}$ levels. The levels of intracellular $\mathrm{Fe}^{2+}$ were increased in A375 and G361 cells following erastin and RSL3 treatment (Figs. 2e, f). Overexpression of miR-137 reduced the levels of intracellular $\mathrm{Fe}^{2+}$ in A375 and G-361 cells following treatment with erastin or RSL3 (Fig. 2e). In contrast, anti-miR-137 increased the levels of intracellular $\mathrm{Fe}^{2+}$ (Fig. 2f). Interestingly, the effect of miR-137 on lipid peroxidation was more significant than iron accumulation $(* * p<0.05$ vs * $p$ $<0.01$ ), which suggests that miR-137 inhibits ferroptosis more effectively through regulating lipid peroxidation in melanoma cells. 
a $\begin{array}{cc}\text { miR-137 } & \text { GAUGCGCAUAAGAAUUCGUUAUU } \\ \text { SLC1A5 3'UTR } & \text { GAGGACAUUUUUUUUAGCAAUAA } \\ \text { Mutant 3'UTR } & \text { GAGGACAUUUUUUUUCAACCAAU }\end{array}$

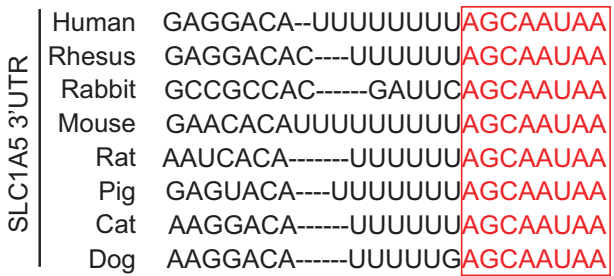

b
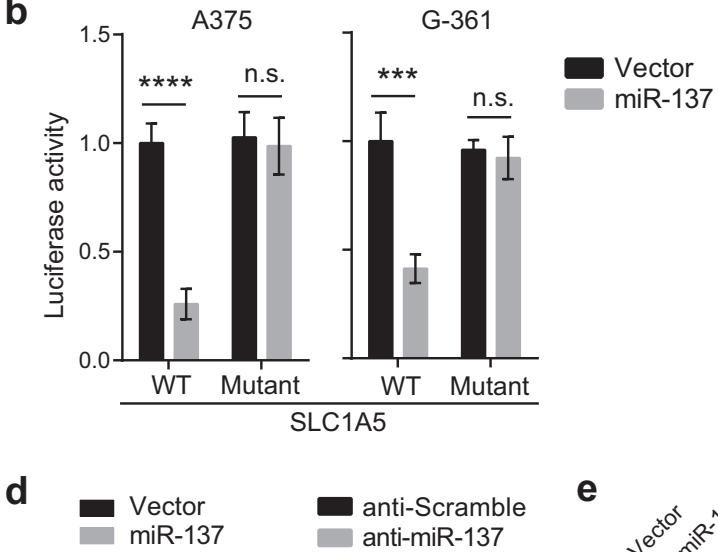

anti-Scramble

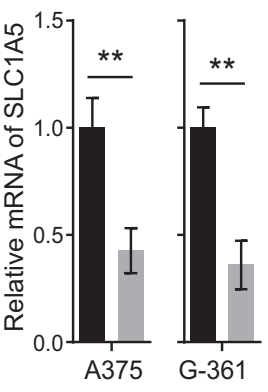
anti-miR-137

g
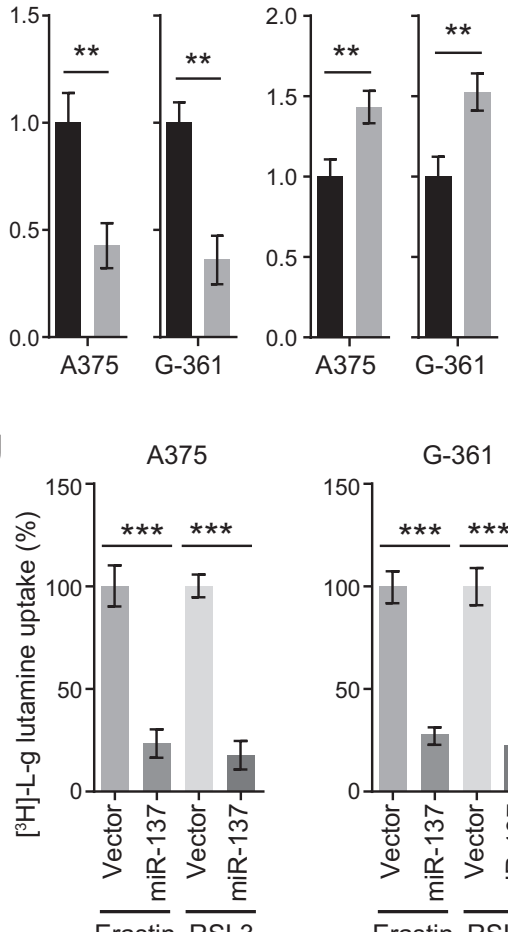

e

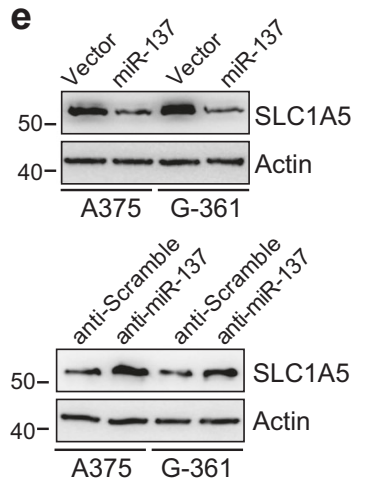

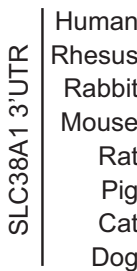

UACAGACCAUCAUUUAGCAAUAC UACAGACCAUCAUUUAGCAAUAC

-----------UUAGUUUGCAAUGUU

C-------U--CCUAGUUAGCAAUGUU

A--CU------AUCAUUUAACAAUGUU

--CUA--U--AUCAUUUAGCAAUGCU

--CUA--C--AUCAUUGAGCAGGGCC

C

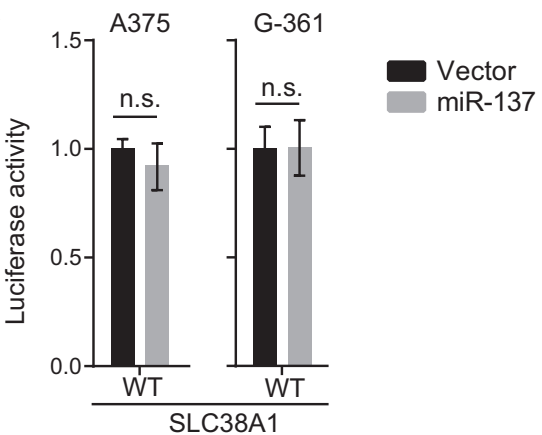

f
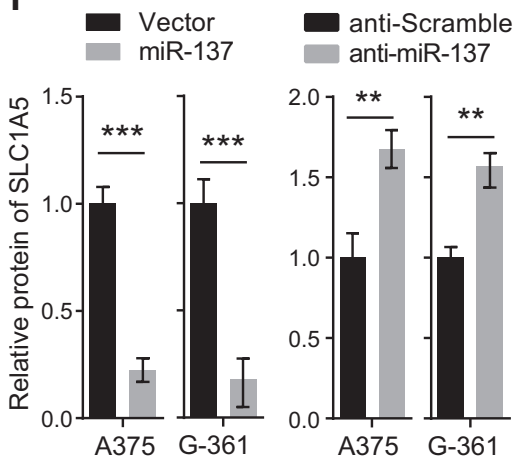

h

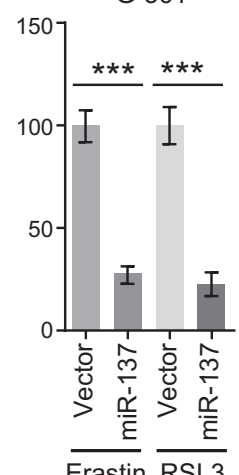

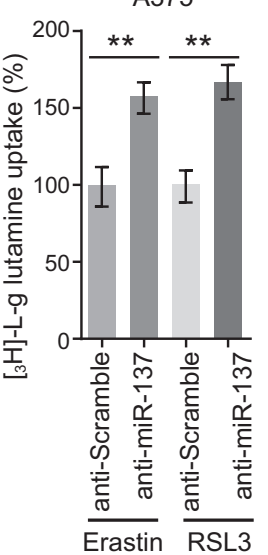

G-361

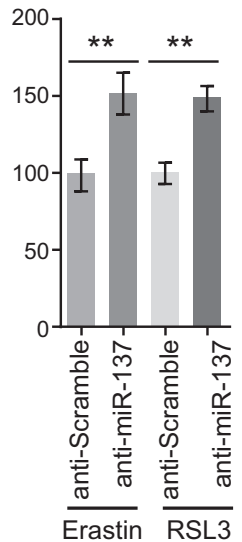


Fig. 4 miR-137 directly targets SLC1A5 in melanoma cells. a Sequence alignment of miR-137 and the $3^{\prime}$-UTR of SLC1A5 or SLC38A1. The seed sequence of miR-137 and the binding sites in 3'UTR are indicated in red. The 3'-UTR mutants containing mismatched nucleotides are shown at the bottom. The binding site of SLC1A5 is highly conserved in several species (left), but the binding site of SLC38A1 is not conserved between different species (right). b miR137 overexpression inhibited the expression of 3'-UTR-luciferase reporter of SLC1A5 in A375 and G-361 cells, but the mutant construct was immune to miR-137. Data are mean \pm SD from three independent experiments. $* * * p<0.001$; n.s. not significant. c miR-137 overexpression has no effect on the expression of $3^{\prime}$-UTR-luciferase reporter of SLC38A1 in A375 and G-361 cells. d miR-137 regulates the mRNA level of SLC1A5 in A375 and G-361 cells. The mRNA levels were measured by quantitative RT-PCR, data are mean \pm SD from three independent experiments. ${ }^{*} p<0.01$. e The protein levels of SLC1A5 were modulated by miR-137 in A375 and G-361 cells. Cells transfected with indicated constructs or antagomirs were collected, and the protein level of SLC1A5 was detected by western blot. Actin was used as a loading control. f Quantitative analysis of the experiments in (e). Data are mean $\pm \mathrm{SD}$ from three independent experiments. ${ }^{*} p<0.01$, $* * * p<0.001$. g miR-137 overexpression significantly suppressed the $\left[{ }^{3} \mathrm{H}\right]$-L-glutamine uptake in A375 and G361 cells. Indicated cells were treated with erastin $(5 \mu \mathrm{M}$ in $\mathrm{A} 375,10$ $\mu \mathrm{M}$ in G-361) or RSL3 (0.1 $\mu \mathrm{M}$ in A375, $0.5 \mu \mathrm{M}$ in G-361) for $24 \mathrm{~h}$. Data shown represent mean \pm SD from three independent experiments. $* * * p<0.001$. h Inhibition of endogenous miR-137 showed increased $\left[{ }^{3} \mathrm{H}\right]-\mathrm{L}-$ glutamine uptake in A375 and G-361 cells. Indicated cells were treated with erastin $(5 \mu \mathrm{M}$ in A375, $10 \mu \mathrm{M}$ in G-361) or RSL3 $(0.1 \mu \mathrm{M}$ in $\mathrm{A} 375,0.5 \mu \mathrm{M}$ in G-361) for $24 \mathrm{~h}$. Data shown represent mean $\pm \mathrm{SD}$ from three independent experiments. ${ }^{* *} p<0.01$

\section{Glutaminolysis is required for miR-137 regulated ferroptosis}

As the most abundant amino acid in the body, cancer cells use Gln both as a nitrogen source for the biosynthesis of nucleotides, amino acid, and lipids as well as an important carbon source for the tricarboxylic acid cycle and cellular energetics [28]. Recent studies have demonstrated that inhibition of glutaminolysis prevents ferroptosis by reducing the formation of oxidizable membrane lipids (Fig. 3a) $[8,10]$. To detect whether miR-137 inhibits ferroptosis through regulating glutaminolysis, we transfected A375 and G-361 cells with anti-miR-137 and treated cells with different pharmacological inhibitors to block Gln metabolism (Fig. 3a). The import of Gln mainly depends on SLC1A5 and SLC38A1, which can be inhibited by L-g-glutamyl-pnitroanilide (GPNA) [29]. As shown in Fig. 3b and Figure S1a, the cell death induced by anti-miR-137 was strongly suppressed by GPNA in erastin-treated A375 and G-361 cells. Similarly, GPNA treatment effectively reduced the accumulation of MDA (Fig. 3b and Fig. S1a).

After import, Gln is converted into Glu by GLS2 [30]. We found that inhibition of GLS2 by compound 968 significantly blocked anti-miR-137-induced cell death and MDA production in A375 cells (Fig. 3b) and G-361 cells
(Fig. S1a). Glu can be further converted into a-KG by GOT1 [31, 32]. We found that AOA, a pan inhibitor of transaminases [33], inhibited both cell death and MDA accumulation in A375 and G-361 cells enhanced by antimiR-137 (Fig. 3b and Fig. S1a). Consistently, RNA interference knockdown of these glutaminolysis genes markedly blocked ferroptosis-related cell death and MDA production in miR-137-knockdown A375 cells (Figs. 3c-e) and G-361 cells (Fig. S1b-d). Thus, both pharmacological and genetic inhibition of the glutaminolysis pathway blocked the induction activity of anti-miR-137 in ferroptosis, suggesting that glutaminolysis is required for miR-137 regulated ferroptosis.

\section{miR-137 directly targets and negatively regulates SLC1A5}

To identify specific targets of miR-137, we searched for glutaminolysis genes containing potential miR-137-binding sites in their $3^{\prime}$-UTRs using publicly available bioinformatics tools TargetScan and miRanda [34-36]. We found that the 3'-UTRs of SLC1A5 and SLC38A1 contain the putative miR-137-binding sites predicted by both algorithms (Fig. 4a). However, the binding site of SLC1A5 is highly conserved in many species, whereas no conservation was observed in the 3'-UTR of SLC38A1 (Fig. 4a). These findings suggest that SLC1A5 might be a direct target of miR-137.

To confirm the bioinformatics-based predictions, we performed luciferase activity assays in A375 and G-361 cells. We first cloned the 3 '-UTR sequences of SLC1A5 and SLC38A1 into the psiCHECK-2 vector. We next introduced several mismatch mutations into the seed sequences to generate mutant reporter vectors. As shown in Fig. 4b, cotransfection of A375 and G-361 cells with miR-137 overexpression constructs dramatically attenuated the luciferase activity of the reporter vector containing the wild-type $3^{\prime}$ UTR of SLC1A5. Although mutation of the seed sequence of miR-137 blocked the inhibitory effect of miR-137 (Fig. 4b). In contrast, miR-137 had no significant effect on the reporter vector containing the wild-type $3^{\prime}$-UTR of SLC38A1 (Fig. 4c). These results indicate that miR-137 directly targets the 3'-UTR region of SLC1A5.

To further investigate whether SLC1A5 is truly a target of miR-137, we next examined the effect of miR-137 on the mRNA and protein levels of SLC1A5. A375 and G-361 cells were transfected with a miR-137 overexpression construct or an antagomir, and the mRNA and protein levels of SLC1A5 were analyzed by quantitative reverse transcriptase-PCR (qRT-PCR) and western blot, respectively. We found that cells transfected with miR-137 overexpression construct caused a significant decrease in both mRNA level and protein level of SLC1A5 (Figs. 4d-f). 
Conversely, treatment with the miR-137 antagomir resulted in increased mRNA and protein expression of SLC1A5 (Figs. 4d-f). However, miR-137 had no significant effect on both mRNA and protein levels of SLC38A1 (Fig. S2a-c). These findings suggest that miR-137 regulates cellular levels of SLC1A5 in melanoma cells.

SLC1A5 is an essential transporter for Gln uptake, miR-137 should modulate the Gln import by regulating SLC1A5. To test this hypothesis, we transfected the cells with miR-137 and measured the Gln uptake rates. As shown in Fig. 4g, the Gln uptake in miR-137 overexpression cells was strongly suppressed compared with the control cells. Conversely, cells transfected with miR-137 antagomir resulted in an increase in Gln uptake in both A375 and G-361 cells (Fig. 4h). Taken together, these results indicate that miR-137 suppresses Gln uptake by targeting SLC1A5.

\section{Overexpression of SLC1A5 restored miR-137- mediated ferroptosis suppression}

To analyze whether miR-137 modulates ferroptosis through its effects on SLC1A5, we performed rescue experiments by overexpressing SLC1A5. The coding sequence of SLC1A5 was cloned into the expressing vector, which did not have the miR-137-binding sequence; it was therefore resistant to miRNA-mediated downregulation. First, cells transfected with miR-137 significantly suppressed erastin-induced cell death in A375 and G-361 cells, but this inhibitory effect was rescued by co-transfected with SLC1A5 overexpression vectors (Figs. 5a, f). In other words, supplement of SLC1A5 protein was sufficient to increase ferroptosis level even in the presence of the miR-137. Second, we observed that the Gin uptake suppressed by miR-137 was strongly induced in SLC1A5 overexpression cells (Figs. 5b, g). Third, the lipid ROS accumulation revealed by MDA assay and BODIPY-C11 staining was also restored upon overexpression of SCL1A5 (Figs. 5c, d, h, i). More importantly, inhibition of SLC1A5 by GPNA can prevent the rescue function of SLC1A5 in both A375 (Figs. 5a-d) and G-361 cells (Figs. 5f-i), which further confirmed SLC1A5 is the target of miR-137 during ferroptosis in melanoma cells. The cellular protein levels of SLC1A5 in different cells were detected by western blot (Figs. 5e, j). These results show that miR-137 regulates ferroptosis by modulating the expression of glutamine transporter SLC1A5 in melanoma.

\section{a-KG enhances ferroptosis in a manner insensitive to $\mathrm{miR}-137$}

Gln plays an important role in ferroptosis, which can enhance cell death and MDA accumulation in A375 and G-
361 cells treated with erastin or RSL3 in a dose-dependent manner (Figs. 6a, b and Fig. S3a, b). As miR-137 suppresses Gln import by targeting SLC1A5, we then tested whether miR-137 can block Gln-induced cell death and MDA accumulation. As expected, Gln did not enhance cell death and MDA accumulation in A375 and G-361 cells transfected with miR-137 (Figs. 6a, b and Fig. S3a,b). Gln can be converted to a-KG through glutaminolysis (Fig. 3a), and $\mathrm{a}-\mathrm{KG}$ can mimic the ferroptosis enhancer activity of Gln [10]. As a downstream metabolite of glutaminolysis, a$\mathrm{KG}$ should rescue the reduction of ferroptosis mediated by the overexpression of miR-137. Indeed, after erastin or RSL3 treatment, a-KG induced both cell death and MDA accumulation in a dose-dependent manner even in the presence of miR-137 (Figs. 6c, d and Fig. S3c,d). These results suggested that the downstream metabolites of glutaminolysis, like a-KG, can enhance ferroptosis insensitive to miR137. This conclusion is also consistent with the finding that miR-137 targets Gln transporter protein SLC1A5, which is upstream of glutaminolysis.

\section{miR-137 suppresses ferroptosis both in vitro and in vivo}

The observed effects of miR-137 on ferroptosis suggest that the expression level of miR-137 should modulate the antitumor activity of erastin and RSL3. Thus, we stably overexpressed miR-137 in A375 cells by lentiviral vector and treated the cells with erastin, and measured its effect on cell viability by colony formation assay. We found that ectopic expression of miR-137 significantly reduced erastininduced ferroptotic cell death (Fig. 7a). Meanwhile, we also used lentiviral vector to stably and specifically knockdown miR-137 by overexpressing the target sequence of miR-137 [37]. As shown in Fig. 7a, knockdown of miR137 markedly increased erastin-induced ferroptotic cell death. The mRNA levels of SLC1A5 in different A375 cells were measured by qRT-PCR, and the results confirmed that miR-137 modulates the expression levels of SLC1A5 (Fig. 7b). Similarly, the expression level of miR-137 can regulate erastin-induced ferroptotic cell death in G-361 cells (Figs. 7c, d), which suggests that the function of how miR137 regulates ferroptosis is not cell specific.

We next investigated whether knockdown of miR-137 enhances erastin-induced ferroptotic cell death in vivo. miR-137 overexpression or knockdown A375 cells were implanted into the subcutaneous space of nude mice. When the tumors reached at $50 \mathrm{~mm}^{3}$, mice were treated with erastin for 20 days. Compared with the control vector group, repression of miR-137 effectively reduced the size of tumors formed (Fig. 7e). In contrast, the size of tumors with ectopic expression of miR-137 is markedly bigger than the control group (Fig. 7e), which suggests that miR-137 

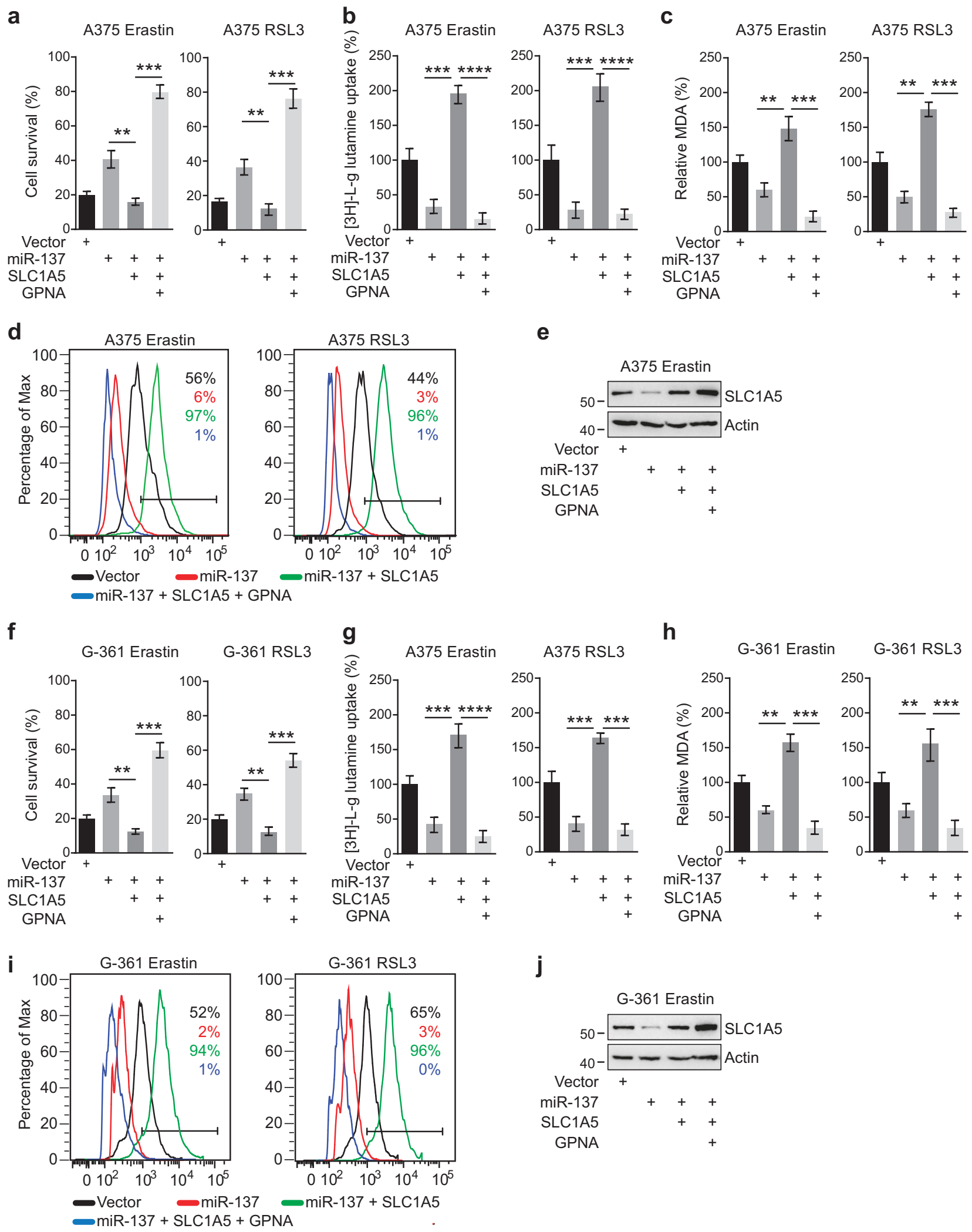

h
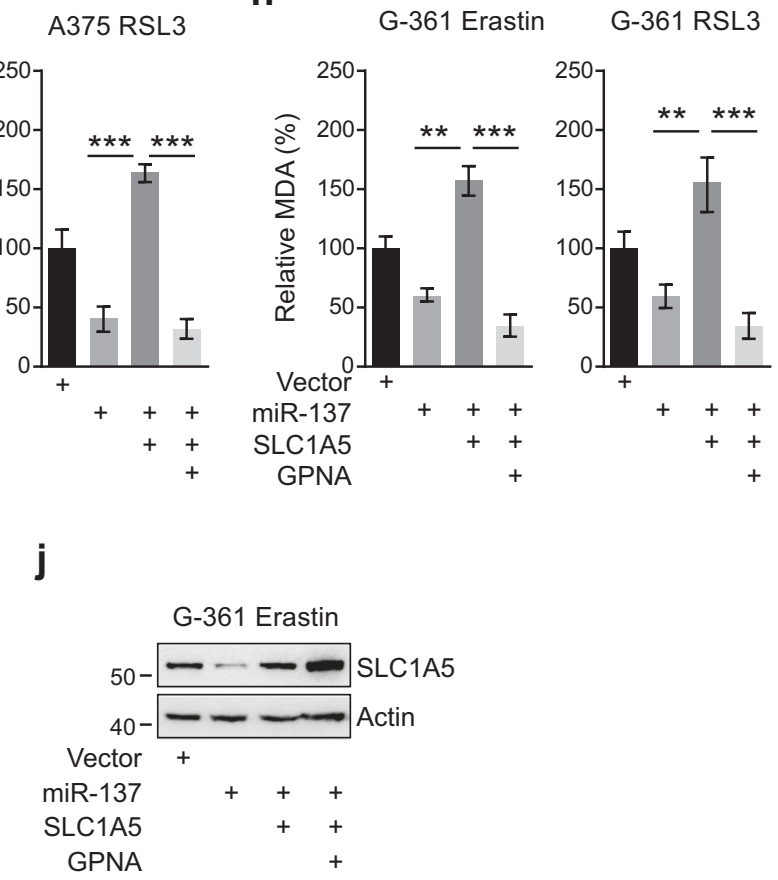

blocked erastin-induced ferroptotic cell death in vivo. Similarly, miR-137 also modulated the antitumor activity of erastin in G-361 cells in mice (Fig. 7f). Collectively, these findings demonstrate that miR-137 plays an critical role in the regulation of ferroptosis cell death in a subcutaneous melanoma mouse model. 
Fig. 5 Overexpression of SLC1A5 rescued miR-137-mediated ferroptosis inhibition. $\mathbf{a}, \mathbf{b}, \mathbf{c}$ Cell viability $\mathbf{a}$, intracellular MDA levels $\mathbf{b}$, and $\left[{ }^{3} \mathrm{H}\right]$-L-glutamine uptake $\mathbf{c}$ of indicated cells were measured. A375 cells were transfected with indicated constructs and treated with erastin $(5 \mu \mathrm{M})$ or RSL3 $(0.1 \mu \mathrm{M})$. The concentration of GPNA is $5 \mathrm{mM}$. Data are mean $\pm \mathrm{SD}$ from three independent experiments. $* * p<0.01$, *** $p$ $<0.001$, $* * * p<0.0001$. d Lipid ROS levels of indicated cells were measured by flow cytometry using C11-BODIPY. A375 cells were transfected with indicated constructs and treated with erastin $(5 \mu \mathrm{M})$ or RSL3 $(0.1 \mu \mathrm{M})$. e Immunoblot analysis of SLC1A5 in A375 cells following indicated transfections and treatments. Actin was used as a loading control. f, $\mathbf{g}, \mathbf{h}$ Cell viability $\mathbf{f}$, intracellular MDA levels $\mathbf{g}$ and $\left[{ }^{3} \mathrm{H}\right]$-L-glutamine uptake $\mathbf{h}$ of indicated cells were measured. G-361 cells were transfected with indicated constructs and treated with erastin $(10 \mu \mathrm{M})$ or RSL3 $(0.5 \mu \mathrm{M})$. The concentration of GPNA is $5 \mathrm{mM}$. Data are mean $\pm \mathrm{SD}$ from three independent experiments. $* * p<0.01$, $* * * p$ $<0.001, * * * * p<0.0001$. i Lipid ROS levels of indicated cells were measured by flow cytometry using C11-BODIPY. G-361 cells were transfected with indicated constructs and treated with erastin $(10 \mu \mathrm{M})$ or RSL3 $(0.5 \mu \mathrm{M})$. j Immunoblot analysis of SLC1A5 in G-361 cells following indicated transfections and treatments. Actin was used as a loading control
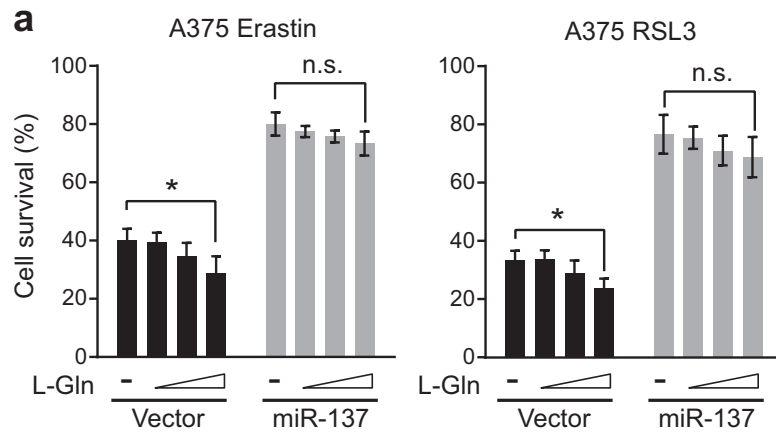

C

A375 Erastin
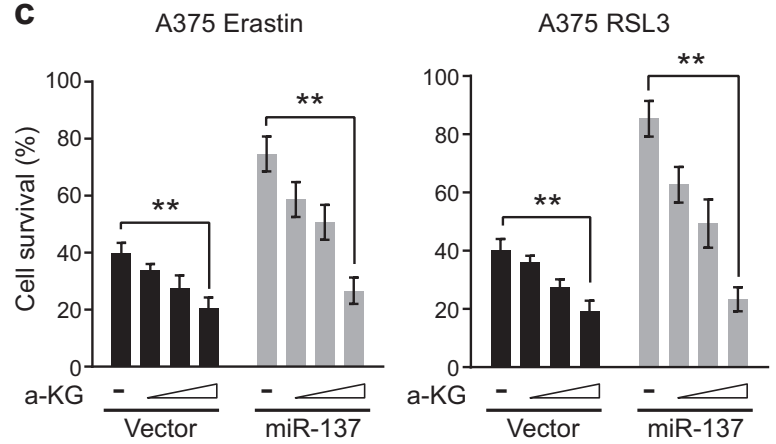

Fig. 6 miR-137-mediated ferroptosis inhibition was rescued by a-KG but not glutamine. a L-Gln induces cell death in erastin or RSL3treated A375 cells in a dose-dependent manner. Overexpression of miR-137 blocked the cell death-inducing activity of L-Gln. Indicated cells were treated with erastin $(5 \mu \mathrm{M})$ or RSL3 $(0.1 \mu \mathrm{M})$ for $24 \mathrm{~h}$, and the cell viability was assayed using a CCK-8 kit. The concentrations of L-Gln were $0.02,0.1$ and $0.5 \mathrm{mM}$. Data are mean \pm SD from three independent experiments. n.s. not significant; ${ }^{*} p<0.05$. b The intercellular MDA levels of erastin or RSL3-treated A375 cells were induced by L-Gln in a dose-dependent manner, which were blocked by the overexpression of miR-137. Indicated cells were treated with erastin $(5 \mu \mathrm{M})$ or RSL3 $(0.1 \mu \mathrm{M})$ for $24 \mathrm{~h}$. The concentrations of L-Gln

\section{Discussion}

miR-137 is an important tumor suppressor. It regulates cancer cell proliferation, migration, apoptosis, and autophagy in many types of cancers by targeting XIAP, FUNDC1, NIX, SRC3, and more [38-40]. In the present study, we introduced miR-137 as the first miRNA that regulates erastin- and RSL3-induced ferroptosis in melanoma (Fig. 7g). miR-137 overexpression caused a significant decrease of Gln transporter SLC1A5. Overexpression of SLC1A5 rescued the ferroptosissuppressing effect of miR-137, confirming that miR-137 modulates ferroptosis through its inhibitory effects on SLC1A5.

SLC1A5 is a cell surface transporter that mediates uptake of neutral amino acids including Gln [41]. The intracellular Gln pool is critical for sustained activation of mammalian target of rapamycin complex 1 (mTORC1) signaling [42], a master regulator of cell growth, apoptosis, and autophagy.

b
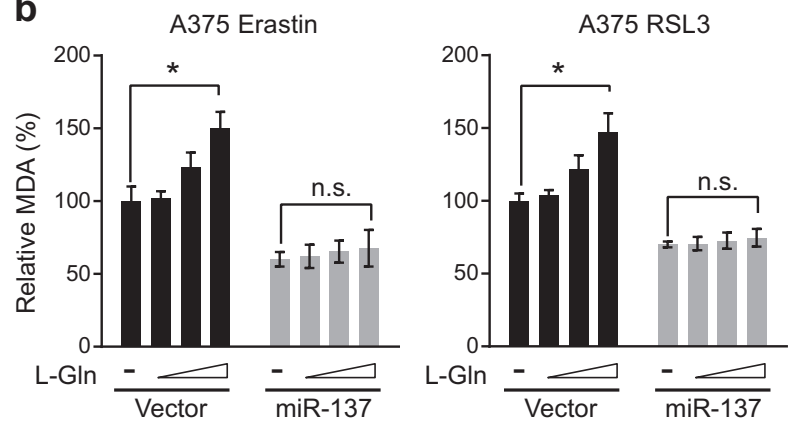

d
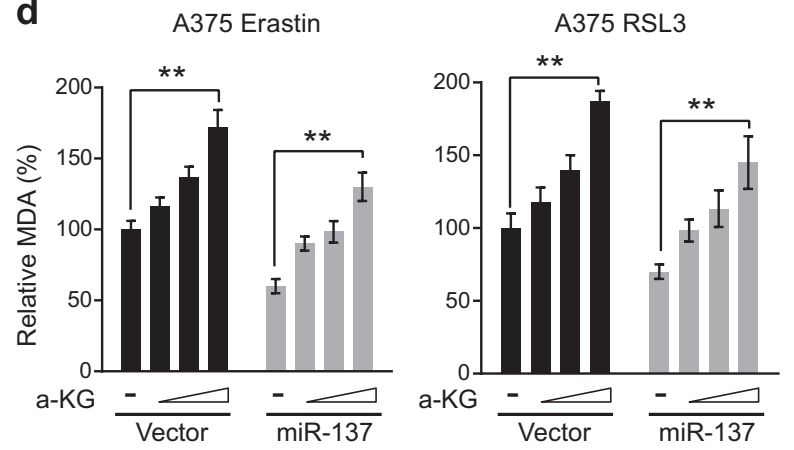

were $0.02,0.1$ and $0.5 \mathrm{mM}$. Data are mean \pm SD from three independent experiments. $* p<0.05$. c a-KG induces cell death in both control and miR-137-overexpressing A375 cells in a dose-dependent manner. Indicated cells were treated with erastin $(5 \mu \mathrm{M})$ or RSL3 $(0.1$ $\mu \mathrm{M})$ for $24 \mathrm{~h}$. The concentrations of a-KG were $0.5,1$ and $2 \mathrm{mM}$. Data are mean \pm SD from three independent experiments. $* * p<0.01$. d The intercellular MDA levels in both control and miR-137-overexpressing A375 cells were induced by $\mathrm{a}-\mathrm{KG}$ in a dose-dependent manner. Indicated cells were treated with erastin $(5 \mu \mathrm{M})$ or RSL3 $(0.1 \mu \mathrm{M})$ for $24 \mathrm{~h}$. The concentrations of a-KG were $0.5,1$ and $2 \mathrm{mM}$. Data are mean $\pm \mathrm{SD}$ from three independent experiments. $* * p<0.01$ 

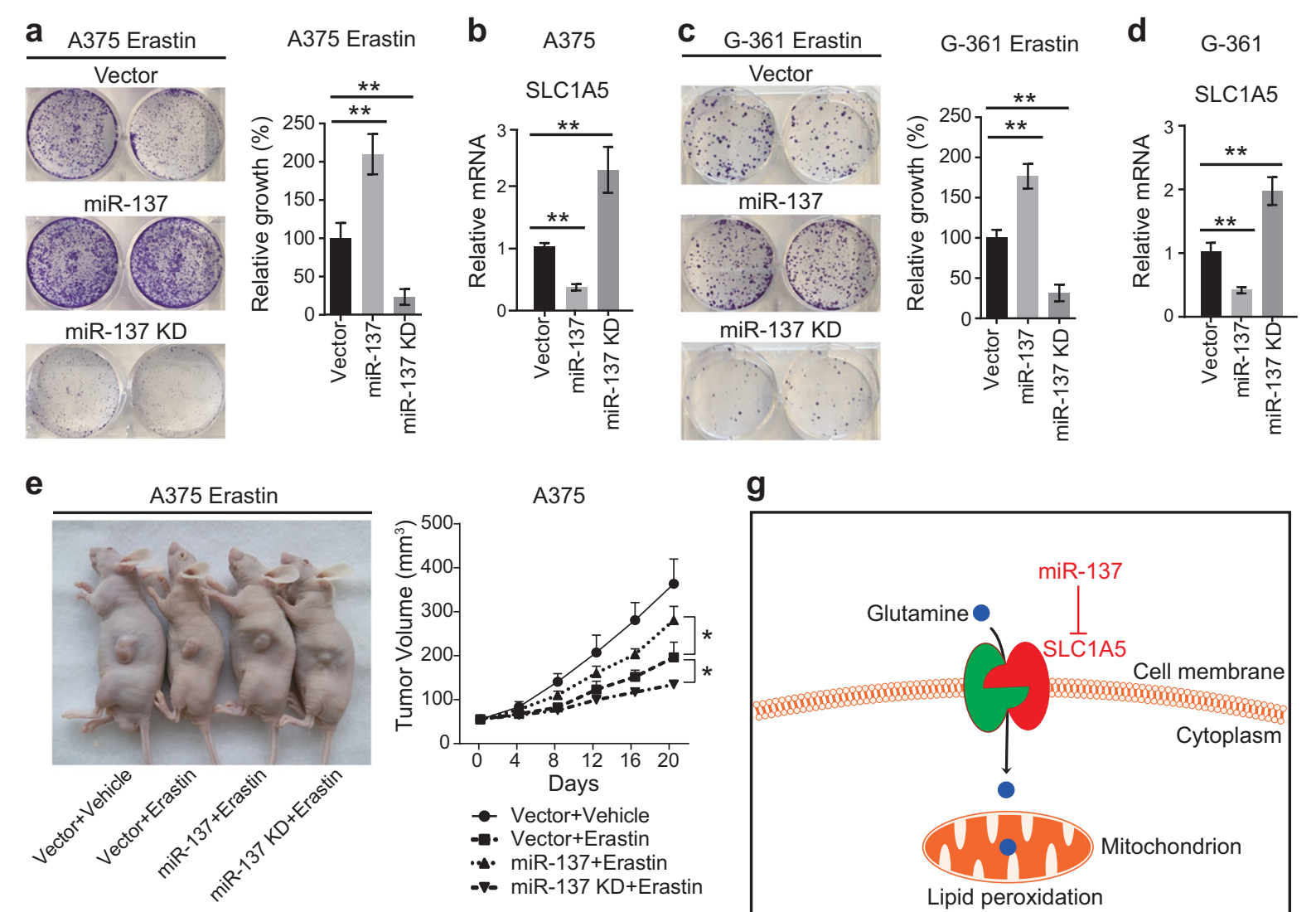

g
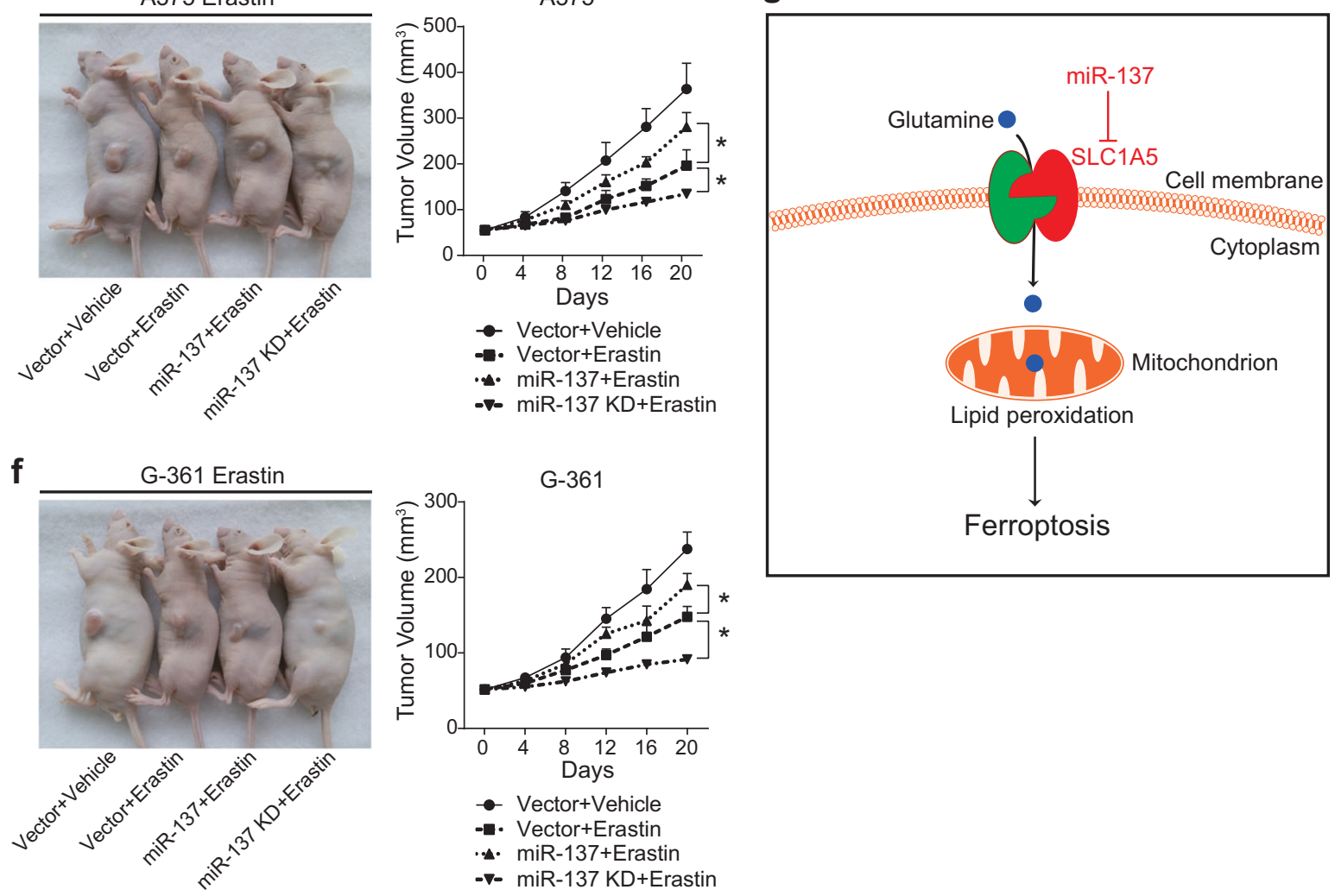

Fig. 7 Inhibition of miR-137 enhances the ferroptosis activity in vivo. a Colony formation assay of the indicated A375 cell lines. Cells were treated with erastin $(5 \mu \mathrm{M})$ for $24 \mathrm{~h}$, and were grown without erastin for 14 days. For each cell line, all dishes were fixed, stained, and photographed at the same time. Data are mean \pm SD from three independent experiments. ${ }^{*} p<0.01$. b Quantitative RT-PCR showing the relative expression levels of SLC1A5 in indicated A375 cells in a. Data are mean $\pm \mathrm{SD}$ from three independent experiments. ${ }^{*} p<$ 0.01. c Colony formation assay of the indicated G-361 cell lines. Cells were treated with erastin $(10 \mu \mathrm{M})$ for $24 \mathrm{~h}$, and were grown without erastin for 14 days. For each cell line, all dishes were fixed, stained, and photographed at the same time. Data are mean \pm SD from three independent experiments. ${ }^{* *} p<0.01$. d Quantitative RT-PCR

In erastin- and RSL3-induced ferroptosis, the Gln importation and metabolism induces lipid ROS generation and promotes cell death [10]. Suppression of SLC1A5 by miR- showing the relative expression levels of SLC1A5 in indicated G-361 cells in c. Data are mean \pm SD from three independent experiments. $* * p<0.01$. e, f Suppression of miR-137 enhances erastin-induced ferroptosis in vivo. C57BL/6 mice were injected subcutaneously with indicated A375 cells e or G-361 cells $\mathbf{f}\left(1 \times 10^{6}\right.$ cells per mouse $)$ and treated with erastin $(15 \mathrm{mg} / \mathrm{kg}$ intraperitoneally, twice every other day) when the tumor volume reached $50 \mathrm{~mm}^{3}$. Tumor volume was calculated every 4 days. Data represent mean \pm SD $(n=5-6$ mice per group). Significance was determined by one-sided Mann-Whitney $U$ test, $* p<0.05$. $\mathrm{g}$ Schematic depicting miR-137-mediated ferroptosis in melanoma cells. miR-137 suppresses erastin-induced ferroptosis by directly targeting Gln importer SLC1A5

137 or small molecular inhibitor GPNA strongly inhibits glutaminolysis to cause ferroptotic cell death. SLC1A5 is required for cell survival and growth in normal conditions, 
but after erastin and RSL3 treatment it facilitates ferroptotic cell death. As SLC1A5-mediated glutamine transport plays a key role in tumor cell metabolism, proliferation, and ferroptosis, it is a potential way for solid tumor treatment to target glutamine transport via SLC1A5 inhibition.

miR-137 has been identified to be significantly downregulated in melanoma compared with adjacent normal tissues [43, 44]. Similar results have been reported in glioblastoma [45], colorectal cancer [46], and non-small cell lung cancer [40]. As a target of miR-137, the expression levels of SLC1A5 are increased in various types of cancer, such as melanoma [47], neuroblastoma [48], and prostate cancer [49]. The negative correlation between miR-137 and SLC1A5 in many different kinds of cancers suggests that SLC1A5 is an important target of miR-137 to inhibit cancer cell growth. The expression level of miR-137 is tightly associated with clinical characteristics, such as TNM stage, ulcer, distal metastasis, and occurrence site [43, 44]. miR137 is not only involved in cancer pathogenesis and progression, but it also regulates drug resistance in many tumors. For example, silencing of miR-137 induces drug resistance by targeting Aurora kinase A (AURKA) in multiple myeloma [50]. Repression of miR-137 significantly inhibits chemosensitivity to paclitaxel and cisplatin in lung cancer by targeting nuclear casein kinase and cyclin-dependent kinase substrate 1 and caspase 3 [51]. Blocking miR-137 conferred oxaliplatin resistance in colon cancer cells by targeting YBX1 [52]. In our study, we found that knockdown of miR-137 sensitized melanoma cells to erastin-induced ferroptosis, which dramatically suppressed the ability of melanoma cells to form colonies in vitro and to develop tumors in a xenograft mouse model in vivo. Therefore, we speculate that the cancer cells with low miR137 expression could be sensitive to erastin-induced ferroptosis, which may serve as a potential and novel therapeutic strategy for cancer treatment.

miRNAs are known to play crucial roles in diverse biological processes including cell proliferation, apoptosis, autophagy, and so on. Intriguingly, some miRNAs are important regulators in the crosstalk between autophagy and apoptosis. As an important tumor suppressor, miR-137 promotes apoptosis in ovarian cancer cells via the regulation of XIAP [39]. Meanwhile, miR-137 inhibits mitophagy via regulation of two mitophagy receptors FUNDC1 and NIX [38]. In this study, we found that miR-137 suppresses ferroptosis by targeting Gln transporter SLC1A5. Collectively, miR-137 is the first identified miRNA that mediates the cross-regulation among apoptosis, autophagy, and ferroptosis. In our screen, we also identified another important ferroptosis associated miRNA, miR-101, which activated erastin- and RSL3-induced ferroptosis by targeting GSH synthesis enzyme Glu-cysteine ligase (data unpublished). Intriguingly, miR-101 represses three important autophagy- associated proteins RAB5A, ATG4D, and STMN1 [53]; and also targets the important anti-apoptotic protein MCL1 [54]. These findings further strengthen the point that a single miRNA can simultaneously modulate apoptosis, autophagy, and ferroptosis. Further efforts to decipher the crucial role of miRNAs in the interplay between apoptosis, autophagy and ferroptosis have profound clinical implications since the evasion of cell death underlies tumorigenesis and drug resistance. Therefore, such efforts are imperative to improve our understanding of miRNAs in tumorigenesis and present a potentially very useful therapeutic strategy for future treatment.

Acknowledgements This work was supported by the Technology Innovation Program of Beijing Institute of Technology and the National Natural Science Foundation of China (81772915) to YY, and the National Natural Science Foundation of China (81502868) and the Natural Science Foundation of Jiangsu Province (BK20150346) to LW and the state key laboratory of pathogen and biosecurity of China (SKLPBS1505) to HW.

Author contributions ML, LW, and KZ performed the experiments and analyzed the data. HW and YL helped with the mice xenograph work. PZ, TG, and WR participated in the data and sample collection. TZ, LG, and DO helped with the manuscript writing. YY designed the experiments, analyzed the data, and wrote the manuscript.

\section{Compliance with ethical standards}

Conflict of interest The authors declare that they have no conflict of interest.

Open Access This article is licensed under a Creative Commons Attribution-NonCommercial-ShareAlike 4.0 International License, which permits any non-commercial use, sharing, adaptation, distribution and reproduction in any medium or format, as long as you give appropriate credit to the original author(s) and the source, provide a link to the Creative Commons license, and indicate if changes were made. If you remix, transform, or build upon this article or a part thereof, you must distribute your contributions under the same license as the original. The images or other third party material in this article are included in the article's Creative Commons license, unless indicated otherwise in a credit line to the material. If material is not included in the article's Creative Commons license and your intended use is not permitted by statutory regulation or exceeds the permitted use, you will need to obtain permission directly from the copyright holder. To view a copy of this license, visit http://creativecommons. org/licenses/by-nc-sa/4.0/.

\section{References}

1. Galluzzi L, Vitale I, Abrams JM, Alnemri ES, Baehrecke EH, Blagosklonny MV, et al. Molecular definitions of cell death subroutines: recommendations of the Nomenclature Committee on Cell Death 2012. Cell Death Differ. 2012;19:107-20.

2. Fuchs Y, Steller H. Programmed cell death in animal development and disease. Cell. 2011;147:742-58.

3. Thompson CB. Apoptosis in the pathogenesis and treatment of disease. Science. 1995;267:1456-62.

4. Vanden Berghe T, Linkermann A, Jouan-Lanhouet S, Walczak H, Vandenabeele P. Regulated necrosis: the expanding network of 
non-apoptotic cell death pathways. Nat Rev Mol Cell Biol. 2014;15:135-47.

5. Blum ES, Abraham MC, Yoshimura S, Lu Y, Shaham S. Control of nonapoptotic developmental cell death in Caenorhabditis elegans by a polyglutamine-repeat protein. Science. 2012;335:970-3.

6. Bergsbaken T, Fink SL, Cookson BT. Pyroptosis: host cell death and inflammation. Nat Rev Microbiol. 2009;7:99-109.

7. Degterev A, Huang Z, Boyce M, Li Y, Jagtap P, Mizushima N, et al. Chemical inhibitor of nonapoptotic cell death with therapeutic potential for ischemic brain injury. Nat Chem Biol. 2005;1:112-9.

8. Dixon SJ, Lemberg KM, Lamprecht MR, Skouta R, Zaitsev EM, Gleason CE, et al. Ferroptosis: an iron-dependent form of nonapoptotic cell death. Cell. 2012;149:1060-72.

9. Yang WS, SriRamaratnam R, Welsch ME, Shimada K, Skouta R, Viswanathan VS, et al. Regulation of ferroptotic cancer cell death by GPX4. Cell. 2014;156:317-31.

10. Gao M, Monian P, Quadri N, Ramasamy R, Jiang X. Glutaminolysis and transferrin regulate ferroptosis. Mol Cell. 2015;59:298-308.

11. Dolma S, Lessnick SL, Hahn WC, Stockwell BR. Identification of genotype-selective antitumor agents using synthetic lethal chemical screening in engineered human tumor cells. Cancer Cell. 2003;3:285-96.

12. Yang WS, Stockwell BR. Synthetic lethal screening identifies compounds activating iron-dependent, nonapoptotic cell death in oncogenic-RAS-harboring cancer cells. Chem Biol. 2008;15:234-45.

13. Yagoda N, von Rechenberg M, Zaganjor E, Bauer AJ, Yang WS, Fridman DJ, et al. RAS-RAF-MEK-dependent oxidative cell death involving voltage-dependent anion channels. Nature. 2007;447:864-8.

14. Brigelius-Flohe R, Maiorino M. Glutathione peroxidases. Biochim Biophys Acta. 2013;1830:3289-303.

15. Yang WS, Stockwell BR. Ferroptosis: death by lipid peroxidation. Trends Cell Biol. 2016;26:165-76.

16. Zhu S, Zhang Q, Sun X, Zeh HJ, Lotze MT, Kang R, et al. HSPA5 regulates ferroptotic cell death in cancer cells. Cancer Res. 2017;77:2064-77.

17. Doll S, Proneth B, Tyurina YY, Panzilius E, Kobayashi S, Ingold I, et al. ACSL4 dictates ferroptosis sensitivity by shaping cellular lipid composition. Nat Chem Biol. 2017;13:91-98.

18. Yuan H, Li X, Zhang X, Kang R, Tang D. CISD1 inhibits ferroptosis by protection against mitochondrial lipid peroxidation. Biochem Biophys Res Commun. 2016;478:838-44.

19. Yang WS, Kim KJ, Gaschler MM, Patel M, Shchepinov MS, Stockwell BR. Peroxidation of polyunsaturated fatty acids by lipoxygenases drives ferroptosis. Proc Natl Acad Sci USA. 2016;113:E4966-4975.

20. Cao JY, Dixon SJ. Mechanisms of ferroptosis. Cell Mol Life Sci. 2016;73:2195-209.

21. Frankel LB, Lund AH. MicroRNA regulation of autophagy. Carcinogenesis. 2012;33:2018-25.

22. Bartel DP. MicroRNAs: genomics, biogenesis, mechanism, and function. Cell. 2004;116:281-97.

23. Lujambio A, Lowe SW. The microcosmos of cancer. Nature. 2012;482:347-55.

24. Carr EL, Kelman A, Wu GS, Gopaul R, Senkevitch E, Aghvanyan $\mathrm{A}$, et al. Glutamine uptake and metabolism are coordinately regulated by ERK/MAPK during $\mathrm{T}$ lymphocyte activation. $\mathrm{J}$ Immunol. 2010;185:1037-44.

25. Wang Q, Bailey CG, Ng C, Tiffen J, Thoeng A, Minhas V, et al. Androgen receptor and nutrient signaling pathways coordinate the demand for increased amino acid transport during prostate cancer progression. Cancer Res. 2011;71:7525-36.
26. Dixon SJ, Lemberg KM, Lamprecht MR, Skouta R, Zaitsev EM, Gleason CE, et al. Ferroptosis: an iron-dependent form of nonapoptotic cell death. Cell. 2012;149:1060-72.

27. Krutzfeldt J, Rajewsky N, Braich R, Rajeev KG, Tuschl T, Manoharan M, et al. Silencing of microRNAs in vivo with 'antagomirs'. Nature. 2005;438:685-9.

28. DeBerardinis RJ, Lum JJ, Hatzivassiliou G, Thompson CB. The biology of cancer: metabolic reprogramming fuels cell growth and proliferation. Cell Metab. 2008;7:11-20.

29. Esslinger CS, Cybulski KA, Rhoderick JF. Ngamma-aryl glutamine analogues as probes of the ASCT2 neutral amino acid transporter binding site. Bioorg Med Chem. 2005;13:1111-8.

30. Curthoys NP, Watford M. Regulation of glutaminase activity and glutamine metabolism. Annu Rev Nutr. 1995;15:133-59.

31. Hensley CT, Wasti AT, DeBerardinis RJ. Glutamine and cancer: cell biology, physiology, and clinical opportunities. J Clin Invest. 2013;123:3678-84.

32. Son J, Lyssiotis CA, Ying H, Wang X, Hua S, Ligorio M, et al. Glutamine supports pancreatic cancer growth through a KRASregulated metabolic pathway. Nature. 2013;496:101-5.

33. Wise DR, DeBerardinis RJ, Mancuso A, Sayed N, Zhang XY, Pfeiffer HK, et al. Myc regulates a transcriptional program that stimulates mitochondrial glutaminolysis and leads to glutamine addiction. Proc Natl Acad Sci USA. 2008;105:18782-7.

34. Agarwal V, Bell GW, Nam JW, Bartel DP. Predicting effective microRNA target sites in mammalian mRNAs. Elife. 2015;4: e05005.

35. John B, Enright AJ, Aravin A, Tuschl T, Sander C, Marks DS. Human microRNA targets. PLoS Biol. 2004;2:e363.

36. Griffiths-Jones S, Saini HK, van Dongen S, Enright AJ. miRBase: tools for microRNA genomics. Nucleic Acids Res. 2008;36 (Database issue):D154-158.

37. Gentner B, Schira G, Giustacchini A, Amendola M, Brown BD, Ponzoni M, et al. Stable knockdown of microRNA in vivo by lentiviral vectors. Nat Methods. 2009;6:63-66.

38. Li W, Zhang X, Zhuang H, Chen HG, Chen Y, Tian W, et al. MicroRNA-137 is a novel hypoxia-responsive microRNA that inhibits mitophagy via regulation of two mitophagy receptors FUNDC1 and NIX. J Biol Chem. 2014;289:10691-701.

39. Li X, Chen W, Zeng W, Wan C, Duan S, Jiang S. microRNA-137 promotes apoptosis in ovarian cancer cells via the regulation of XIAP. Br J Cancer. 2017;116:66-76.

40. Chen R, Zhang Y, Zhang C, Wu H, Yang S. miR-137 inhibits the proliferation of human non-small cell lung cancer cells by targeting SRC3. Oncol Lett. 2017;13:3905-11.

41. Kanai Y, Clemencon B, Simonin A, Leuenberger M, Lochner M, Weisstanner $M$, et al. The SLC1 high-affinity glutamate and neutral amino acid transporter family. Mol Asp Med. 2013;34:108-20.

42. Duran RV, Oppliger W, Robitaille AM, Heiserich L, Skendaj R, Gottlieb E, et al. Glutaminolysis activates Rag-mTORC1 signaling. Mol Cell. 2012;47:349-58.

43. Bian D, Shi W, Shao Y, Li P, Song G. Long non-coding RNA GAS5 inhibits tumorigenesis via miR-137 in melanoma. Am J Transl Res. 2017;9:1509-20.

44. Li N. Low expression of Mir-137 predicts poor prognosis in cutaneous melanoma patients. Med Sci Monit. 2016;22:140-4.

45. Ciafre SA, Galardi S, Mangiola A, Ferracin M, Liu CG, Sabatino $\mathrm{G}$, et al. Extensive modulation of a set of microRNAs in primary glioblastoma. Biochem Biophys Res Commun. 2005;334:1351-8.

46. Balaguer F, Link A, Lozano JJ, Cuatrecasas M, Nagasaka T, Boland CR, et al. Epigenetic silencing of miR-137 is an early event in colorectal carcinogenesis. Cancer Res. 2010;70:6609-18.

47. Wang Q, Beaumont KA, Otte NJ, Font J, Bailey CG, van Geldermalsen $\mathrm{M}$, et al. Targeting glutamine transport to suppress melanoma cell growth. Int J Cancer. 2014;135:1060-71. 
48. Ren P, Yue M, Xiao D, Xiu R, Gan L, Liu H, et al. ATF4 and NMyc coordinate glutamine metabolism in MYCN-amplified neuroblastoma cells through ASCT2 activation. J Pathol. 2015;235:90-100.

49. Wang Q, Hardie RA, Hoy AJ, van Geldermalsen M, Gao D, Fazli L, et al. Targeting ASCT2-mediated glutamine uptake blocks prostate cancer growth and tumour development. J Pathol. 2015;236:278-89.

50. Qin Y, Zhang S, Deng S, An G, Qin X, Li F, et al. Epigenetic silencing of miR-137 induces drug resistance and chromosomal instability by targeting AURKA in multiple myeloma. Leukemia. 2017;31:1123-35.

51. Shen H, Wang L, Ge X, Jiang CF, Shi ZM, Li DM, et al. MicroRNA-137 inhibits tumor growth and sensitizes chemosensitivity to paclitaxel and cisplatin in lung cancer. Oncotarget. 2016;7:20728-42.

52. Guo Y, Pang Y, Gao X, Zhao M, Zhang X, Zhang H, et al. MicroRNA-137 chemosensitizes colon cancer cells to the chemotherapeutic drug oxaliplatin (OXA) by targeting YBX1. Cancer Biomark. 2017;18:1-9.

53. Frankel LB, Wen J, Lees M, Hoyer-Hansen M, Farkas T, Krogh A, et al. microRNA-101 is a potent inhibitor of autophagy. EMBO J. 2011;30:4628-41.

54. Su H, Yang JR, Xu T, Huang J, Xu L, Yuan Y, et al. MicroRNA101, down-regulated in hepatocellular carcinoma, promotes apoptosis and suppresses tumorigenicity. Cancer Res. 2009;69:1135-42. 